\title{
An experimental study of transient effects in the breakup of viscous drops
}

\author{
By H. A. STONE, B. J. BENTLEY†AND L. G. LEAL \\ Chemical Engineering Department, California Institute of Technology, \\ Pasadena, CA 91125, USA
}

(Received 19 March 1986)

A computer-controlled four-roll mill is used to examine two transient modes of deformation of a liquid drop: elongation in a steady flow and interfacial-tensiondriven motion which occurs after the flow is stopped abruptly. For modest extensions, drop breakup does not occur with the flow on, but may occur following cessation of the flow as a result of deterministic motions associated with internal pressure gradients established by capillary forces. These relaxation and breakup phenomena depend on the initial drop shape and the relative viscosities of the two fluids. Capillary-wave instabilities at the fluid-fluid interface are observed only for highly elongated drops. This study is a natural extension of G. I. Taylor's original studies of the deformation and burst of droplets in well-defined flow fields.

\section{Introduction}

Basic research on the deformation and breakup of a liquid drop due to the motion of an immiscible, viscous suspending fluid dates back to the pioneering work of G. I. Taylor $(1932,1934,1964)$. The problem is of considerable fundamental interest in fluid mechanics as an example of a time-dependent free-boundary problem and as a prototype for flow-induced deformation of a variety of flexible bodies such as red blood cells, macromolecules, flocs, elastic particles, etc. It is also closely related to dispersion processes in commercial blenders and mechanical emulsifiers. As a consequence, many investigations have appeared since Taylor's original papers. Two excellent reviews by Acrivos (1983) and Rallison (1984) provide a comprehensive description of this more recent work.

The remarkable feature of Taylor's early investigations is that he actually discovered most of the interesting phenomena that are characteristic of drop deformation and breakup in steady flows. However, to stop at this point in a description of Taylor's contribution to the drop-deformation and breakup problem does not do justice to the importance of his work. To experimentally simulate a planar extensional flow, Taylor invented the four-roll mill, which has subsequently been used in many laboratories for studies of drop breakup, extension of macromolecules, floc stability, and many related topics. To provide a theoretical description of small deformations of the drop, Taylor used the asymptotic method of domain perturbations. Later, to describe the motion of the highly elongated shapes characteristic of low-viscosity drops, Taylor pioneered slender-body theory for lowReynolds-number flow.

Taylor's (1934) experimental observations in steady simple shear and planar

† Current address: Bentley Systems, Ine., 180 Gordon Dr., Lionville, PA 19353, USA. 
extensional flows show clearly that drops at first deform under the combined action of viscous and pressure forces through a series of steady shapes as the shear rate is increased, until finally a point of maximum steady deformation is reached beyond which further increases in the shear rate usually lead to a transient, steadily increasing extension of the drop with time. As Taylor was first to note, however, there is a fundamental difference between simple shear flow and two-dimensional extensional flow. In particular, a drop with a viscosity that exceeds the suspending fluid viscosity by more than approximately a factor of 4 does not become stretched in simple shear flow, but rather achieves a steady, slightly deformed shape for all large shear rates. Thus, at low Reynolds number, it is impossible to break a sufficiently viscous drop in steady, simple shear flow regardless how large the shear rate becomes. In two-dimensional extensional flow, on the other hand, drops of any viscosity ratio extend continuously so long as the strain rate exceeds a certain critical value. This fundamental distinction, first discovered by Taylor, between simple shear flow and planar extensional flow is of considerable practical importance despite the restriction to low Reynolds numbers, simply because the drops that we attempt to break in blending devices are frequently characterized by low Reynolds numbers.

In spite of the major accomplishments of Taylor and subsequent researchers over the intervening 50 years, however, many important qualitative questions remain to be answered:

(a) What is the role of flow type in the deformation and breakup processes? In particular, what is the nature of the transition between simple shear and twodimensional extensional flows?

(b) What are the mechanisms for breakup and how do they depend on the parameters of the system, including the degree of deformation of the drop?

(c) What is the role of flow transients in the deformation and breakup processes?

(d) What is the role of rheology if one or both fluids are non-Newtonian?

The research reported here and in two earlier publications from our laboratory (Bentley \& Leal $1986 a, b$ ) represent the first steps in a series of experimental (and theoretical) studies that are designed to address these and related questions. These experiments are based upon a computer-controlled version of Taylor's four-roll mill designed to maintain the drop at the stagnation point of the flow, with minimal disturbance, for either steady or unsteady flows. The complete spectrum of linear, two-dimensional flows can be generated from pure rotation to two-dimensional straining flow (the latter being the only motion studied by Taylor with the four-roll mill). A complete description of this device was published in our earlier paper, Bentley \& Leal (1986a).

In our initial investigation, Bentley \& Leal (1986b), we studied steady-state shape and orientation for drops in five different types of steady two-dimensional flows, from pure extension ('hyperbolic flow') to a flow with only slightly more strain than vorticity and for drops with viscosity ranging from $10^{-3}$ to $10^{2}$ that of the suspending fluid. Critical conditions for breakup were also determined for all these cases, the critical conditions being identified as the Capillary number (dimensionless shear rate) beyond which the drop undergoes continuous extension. The Bentley-Leal study of drop deformation in steady flows was a natural outgrowth of preceding research in the field and concentrated mainly on the measurement and prediction of steady shapes and of conditions where steady shapes could not be achieved in steady flows.

The present paper represents a first step toward generalization of preceding studies to investigate transient effects on the deformation and breakup of a liquid drop. In particular, we examine the continuous elongation of a liquid drop for steady 

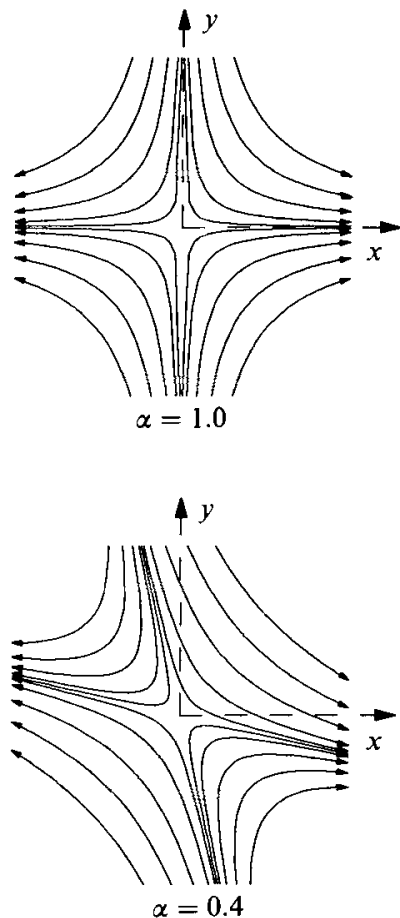
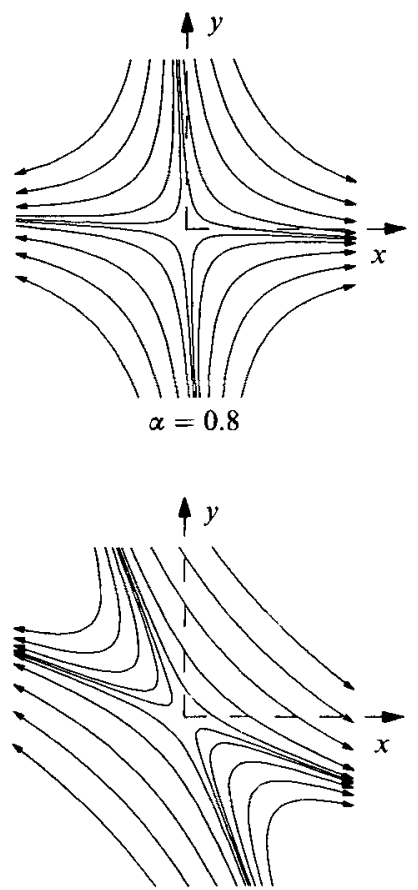

$\alpha=0.2$
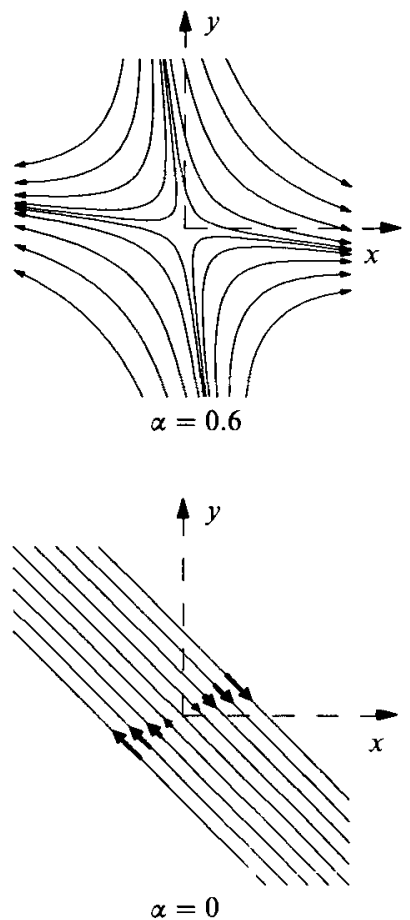

Figure 1. Streamlines for the linear flow $u=\Gamma \cdot x, \Gamma$ given by $(1) ; \alpha \geqslant 0$.

two-dimensional flows at capillary numbers (shear rates) that are equal to or slightly above the critical value for 'breakup', and, the subsequent interfacial-tension-driven relaxation of the extended drop when the flow is stopped abruptly. Relatively few previous studies have examined any aspect of time-dependent behaviour in the deformation and breakup process. Capillary-wave instability on infinite, stationary fluid cylinders has been considered by Tomotika (1935), Rumscheidt \& Mason (1961), Lee \& Flumerfelt (1981) and Lee, Yu \& Flumerfelt (1981), while Tomotika (1936) and Mikami, Cox \& Mason (1975) have studied capillary-wave growth on extending fluid threads of infinite length. Of course, extended drops differ from an infinite cylinder in the sense that they are always closed at their ends, and thus do not represent a possible equilibrium state. We shall see that the final drop length plays a critical role in both the extension of the drop and its relaxation when the flow is removed, with the result that capillary-wave instability plays a role only if the droplets are extremely elongated. The closest experimental study to that reported here is due to Grace (1971), who reports data on the elongation necessary to achieve rupture in the relaxation process, the resulting drop-size distribution, and some interesting effects due to abrupt changes in shear rate for simple shear and planar extensional flows. Torza, Cox \& Mason (1972) also investigated experimentally some effects of the time history of the flow field. From a theoretical point of view, three types of analysis have been used to study transient phenomena. Hinch \& Acrivos (1980) and Hinch (1980) used slender-body theory to investigate the behaviour of low-viscosity drops, and found that the equilibrium shapes corresponding to subcritical shear rates were accessible only if the shear rate was increased slowly. It 


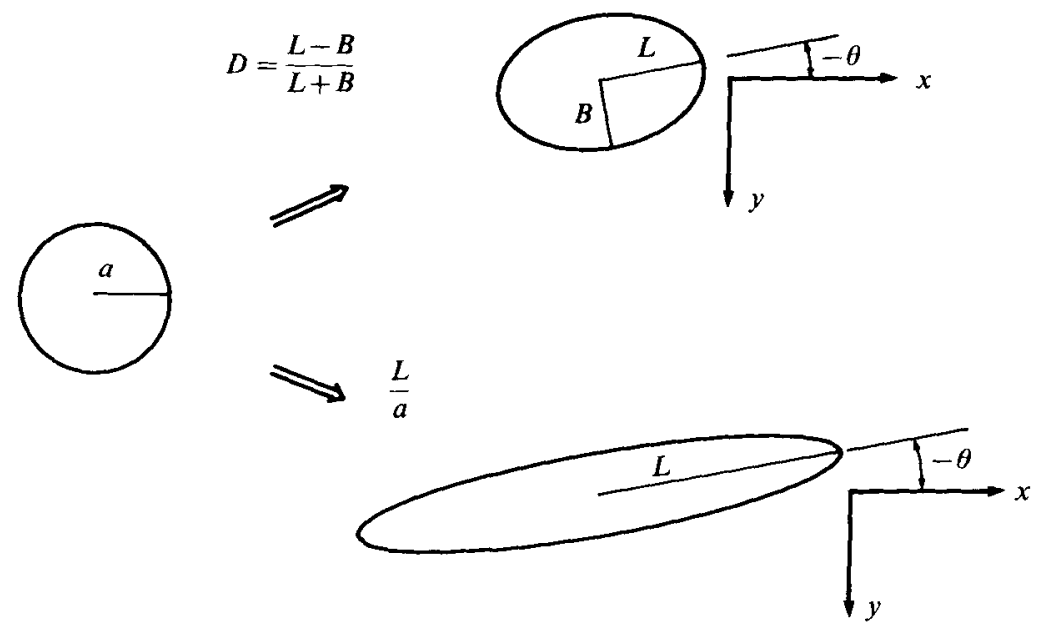

Figure 2. Scalar measures of deformation and orientation.

was also shown that the existence of a waist in the initial shape always led to breakup. Nearly spherical drops of arbitrary viscosity ratio were studied via domain perturbation techniques by Cox (1969) and Rallison (1980), and shown to attain steady shapes in an oscillatory manner in flows with vorticity. Finally, Rallison \& Acrivos (1978) and Rallison (1981) have used the boundary-integral method to investigate some aspects of the time evolution of the drop shape and observed that the critical shear rate and mode of drop burst depended on the history of the flow.

\section{Problem statement}

We consider a Newtonian-liquid droplet, with undeformed radius $a$, density $\hat{\rho}$, and viscosity $\hat{\mu}$, suspended in a second immiscible Newtonian fluid of density $\rho$ and viscosity $\mu$ which is undergoing a linear, two-dimensional flow at infinity. The undisturbed velocity field is $\boldsymbol{u}=\boldsymbol{\Gamma} \cdot \boldsymbol{x}$, where the velocity gradient tensor $\boldsymbol{\Gamma}$ is

$$
\Gamma=\frac{1}{2} G\left[\begin{array}{lll}
1+\alpha & 1-\alpha & 0 \\
-1+\alpha & -1-\alpha & 0 \\
0 & 0 & 0
\end{array}\right]
$$

with $\alpha$ being a 'How-type' parameter and $G$ the fluid shear rate. An approximation to this flow field is generated near the centre of a four-roll mill. The flow-type parameter $\alpha$ provides a measure of the ratio of the rate of strain relative to the vorticity in the undisturbed flow and varies between $\alpha=+1.0$, a hyperbolic (or extensional) flow, and $\alpha=-1.0$, a purely rotational flow. Simple shear flow corresponds to $\alpha=0$. We shall be interested in flows with $\alpha>0$, the so-called 'strong' flows, as they are capable of producing the greatest deformation for a given value of $G$. The streamlines for several of these flows are illustrated in figure 1 . The fluid-fluid interface is characterized by a constant interfacial tension $\sigma$.

Provided that the Reynolds number $\rho G a^{2} / \mu$ is sufficiently small, the behaviour of a neutrally buoyant drop in a steady flow can be characterized by three dimensionless parameters: the viscosity ratio $\lambda=\hat{\mu} / \mu$, the capillary number $\mathbb{C}=\mu G a / \sigma$ (which provides a measure of viscous forces causing deformation relative to interfacial-tension forces which resist deformation), and the flow-type parameter $\alpha$. The orientation of the drop relative to the principal axis of strain (in our device, this is the $x$-axis for 
all flow types) is denoted by $\theta$ and is sketched in figure 2. Two scalar deformation measures have been used in drop-deformation studies and are also shown in figure 2. These are : $D=L-B /(L+B)$, where $L$ and $B$ are the half-length and half-breadth of the drop, respectively, which is appropriate for mildly deformed drops; and $L / a$, the elongation ratio, which is appropriate for highly extended drops. As discussed by Bentley \& Leal $(\mathbf{1 9 8 6} b)$, the orientation angle is important because the effectiveness of a particular flow in deforming the drop depends on the orientation of the drop relative to the principal axis of strain of $\boldsymbol{\Gamma}$. This same concept will arise later when we consider the transient elongation of drops in steady flows characterized by different flow types.

For sufficiently small values of the capillary number $\mathbb{C}$ a steady drop shape exists in a steady two-dimensional flow for all $\alpha$ and $\lambda$. Indeed, for each flow type $\alpha \neq 1$, there exists a viscosity ratio above which the drop attains a steady shape for any value of the capillary number (provided, of course, that the Reynolds number remains small). In such cases, drop burst is impossible. In the majority of cases, though, there exists a critical capillary number above which a steady drop shape no longer exists and viscous forces cause the drop to continually elongate. This transient deformation is examined in the present study. If at some time during this elongation process the flow is stopped, the problem becomes that of an extended liquid drop suspended in a fluid that is otherwise quiescent. Because the extended drop is not an equilibrium shape, an evolutionary, interfacial-tension-driven flow occurs and the drop rapidly changes shape, either returning to a sphere or breaking into smaller drops via a complicated, time-dependent motion. The entire dynamics of this interfacial-tension-driven flow are characterized by the viscosity ratio and the initial drop shape, and this includes the determination of whether the drop breaks or returns to its native spherical shape. Interfacial tension determines the velocity scale for the drop's motion, and hence the timescale of the relaxation or breakup process, but has no role in determining the qualitative characteristics of the phenomena. In the present study we examine this evolution of extended drops as a function of both viscosity ratio and initial elongation ratio $L / a$ at the time flow is stopped. One objective is to determine the elongation ratio that is necessary if the drop is to break, including any dependence of this critical condition on the viscosity ratio or the flow type during the elongation process (the latter presumably affecting the initial elongated shape).

\section{The experiment}

The experiments reported in this paper were performed using the computercontrolled four-roll mill described in Bentley \& Leal (1986a). Here, we discuss only those features of the device that are important to the present work.

The drop position in the device is sensed using a digital television camera. A mini-computer uses the digital representation of the drop to determine the location of its centre of mass. A control scheme which models the response of the flow to changes in roller speed and the drop's response to changes in the flow field then regulates the speeds of the rollers in order to maintain the drop at the centre of the device, under the constraint that the shear rate $G$ and flow type $\alpha$ either remain constant or change in a proscribed manner with time. Typically, about five control cycles occur each second.

Since the drop position is controlled via small but finite modifications of the flow several times per second, an obvious question is whether these changes introduce any 


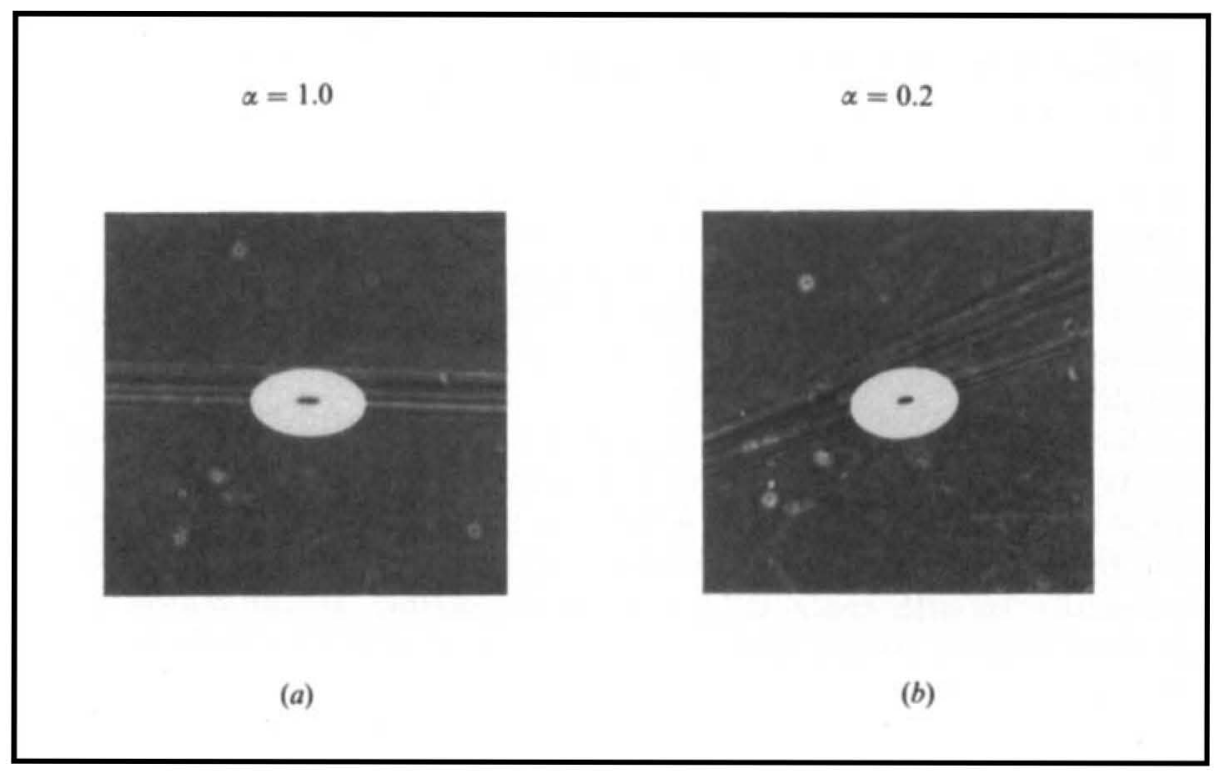

FIGURE 3. Eight second time-exposure photographs of drops in the four-roll mill. The sharpness of the fluid-fluid interface demonstrates the ability of the control scheme to maintain the drop at the device centre with minimal disturbances $(a) \lambda=1.3, \alpha=1.0, G=0.11 \mathrm{~s}^{-1}$. (b) $\lambda=1.3, \alpha=0.2$, $G=0.165 \mathrm{~s}^{-1}$.

discernible change in the shape of the drop. A qualitative indication of the success of the control scheme is illustrated by the sharp fluid-fluid interfaces shown in the time-exposure photographs of figure 3. Photograph $3(a)$ is an $8 \mathrm{~s}$ exposure (approximately 40 control cycles) of a drop at $G=0.11 \mathrm{~s}^{-1}, \alpha=1.0$, while $3(b)$ is an $8 \mathrm{~s}$ exposure with $G=0.165 \mathrm{~s}^{-1}, \alpha=0.2$. The different light intensity at the centre is due the lense effect of the drop. Previous four-roll mills, including Taylor's original device, could be controlled only by manual manipulation of the roller speeds. This resulted in strong, time-dependent changes in the flow, and restricted the studies to steady, hyperbolic flow, $\alpha=1.0$, only. The present apparatus dramatically increases the reliability and utility of the four-roll mill by reducing the flow disturbances to a minimal level and by allowing the full range of both steady and time-dependent two-dimensional flows to be performed.

In our previous studies, the deformation and deformation history of the drop was followed via $35 \mathrm{~mm}$ photographs. However, this procedure is demanding of operator time and is limited in the range of time-dependent motions that can be followed (the minimum time increment between successive photographs for a Canon A-1 motordriven camera is approximately $0.8 \mathrm{~s}$ ). Furthermore, it is expensive with regard to film and developing costs. Thus, one change introduced for the present study is the development of a technique for direct analysis of the existing digital image of the drop to determine the degree of drop deformation.

\subsection{Data analysis using digital image processing}

Digital image-processing techniques have found a wide variety of uses in experimental fluid mechanies. However, so far as we are aware, the only utilization similar to that described below is due to Girault, Schiffrin \& Smith $(1982,1984)$ who used a video digitizing technique to determine the shapes of stationary pendant drops for the measurement of interfacial tension. 


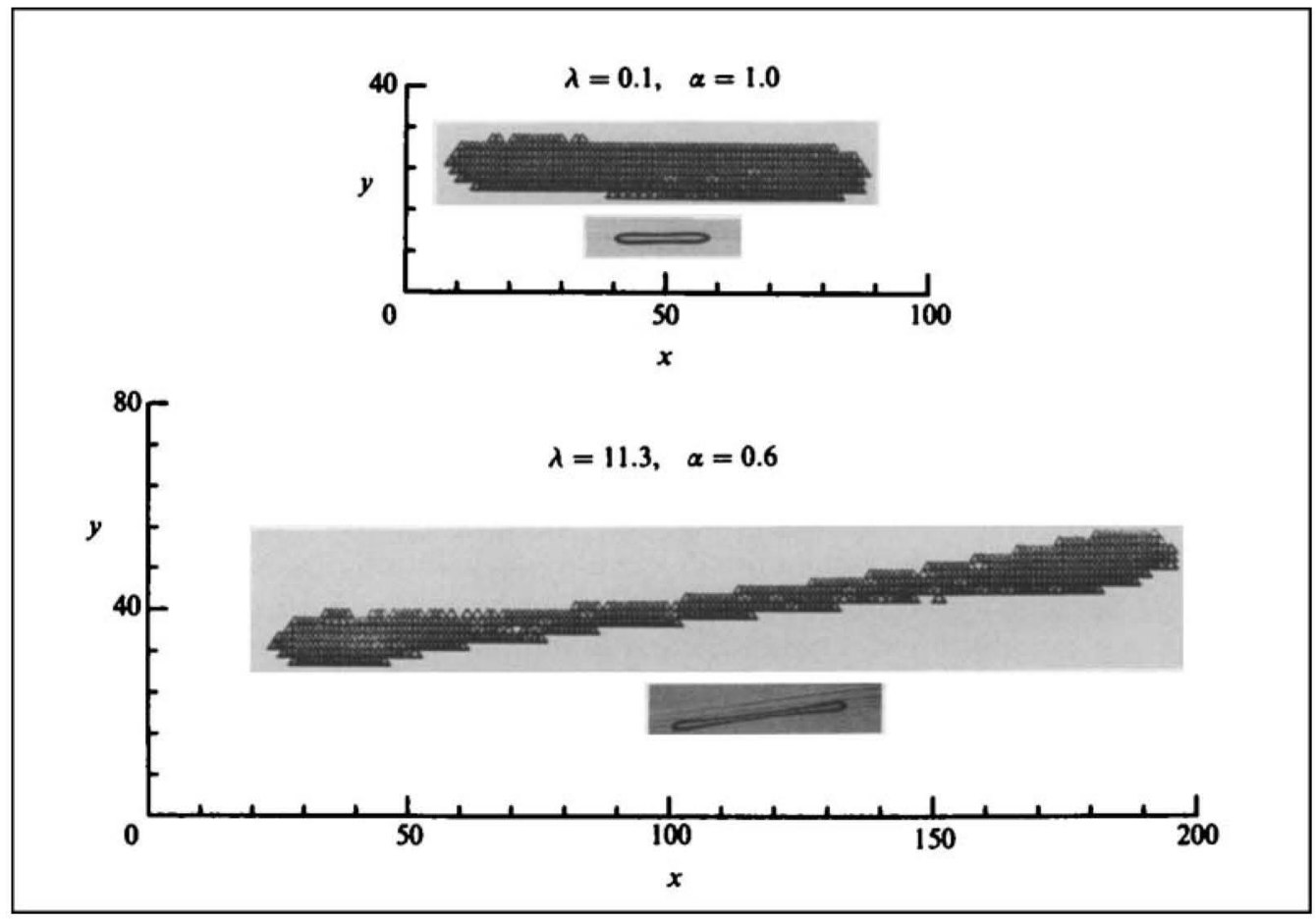

Figure 4. Typical digital images of the transient deformation of highly elongated droplets in a steady flow at the critical capillary number. $35 \mathrm{~mm}$ photographs are shown for comparison.

In our experiment, the sensor of the digital television camera effectively consists of a $224 \times 112$ grid of discrete picture elements (pixels), the light intensity at each pixel being represented by an eight-bit digital signal. This grey level information is thresholded by hardware to a one-bit (or two-state) signal, effectively replacing the entire grey level image by a black drop in a white background. The thresholded information is sent via direct memory access to the computer's memory and used in the control scheme to rapidly determine the position of the centre of mass of the drop. In our previous studies, this was the sole use of the thresholded image. In the present work, however, the thresholded digital image is saved in a data file at the command of the operator, and then analysed, following completion of the experiment, to determine the characteristic scalar measure of the degree of drop deformation; i.e. $D$ or $L / a$. During rapid deformation, many images could be saved, if desired.

The resolution of the digital television camera is 110.2 pixels $/ \mathrm{cm}$ in the $x$-direction and $72.5 \mathrm{pixels} / \mathrm{cm}$ in the $y$-direction (directions are shown in figures 1 and 2 ). The undeformed radius of the drop was typically $0.1 \mathrm{~cm}$, but during the elongation and breakup experiments the drops often attained half-lengths of approximately $1 \mathrm{~cm}$ with very narrow waists, approximately $0.02 \mathrm{~cm}$ in diameter. The narrow cylindrical waist was often very difficult for the digital camera to resolve.

Two digital images of deforming drops are shown in figure 4, along with corresponding still photographs. These images are typical of the transient deformation that is the topic of interest of this paper. The basic features of the shape are captured by the digital image. Following determination of the centre of mass, we calculated $L / a$ by determining the distance separating the farthest edge bit from the centre of mass. Edge bits were located by scanning the image, from the outside toward the inside, until we found the first bit on each scanning line such that two out of three 


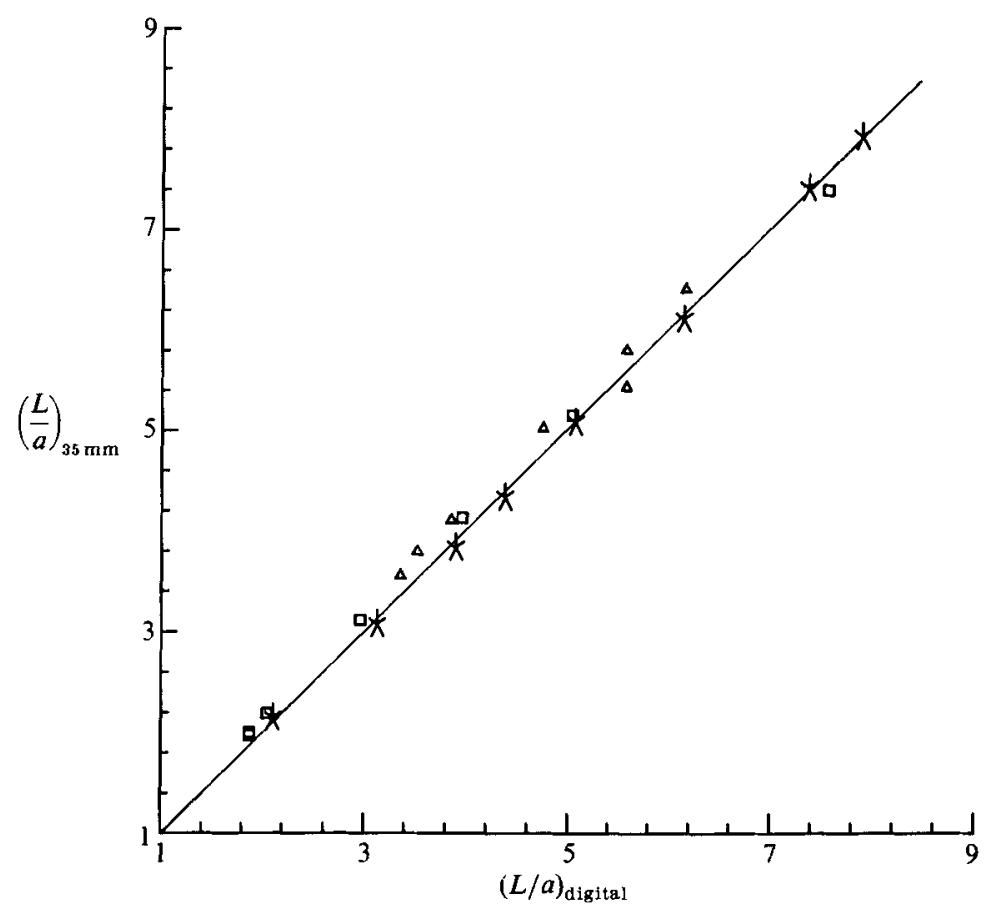

Fraure 5. Comparison of $L / a$ calculated from $35 \mathrm{~mm}$ photographs and $L / a$ calculated by analysing digital images. The solid line denotes $(L / a)_{35 \mathrm{~mm}}=(L / a)_{\text {digital }} ; \triangle, \lambda=0.01, \alpha=1.0 ; \square, \lambda=0.09$, $\alpha=0.4 ; \star, \lambda=12.2, \alpha=1.0$.

successive bits were 'dark'. Such a screening process was necessary to be sure that extraneous dark bits due to 'noise' in the digital image were not inadvertently identified as edge bits. A representative comparison of data from $35 \mathrm{~mm}$ still photographs with the results of analysing the digital image in this manner is shown in figure 5. Results are presented for three different viscosity ratios and two different flow types for a series of experiments on the transient elongation of a liquid droplet, described in further detail in §3.2. Agreement is typically to within $5 \% \dagger$. With this level of accuracy, the digital image-analysis procedure was adopted and used extensively for determination of the degree of drop deformation in the present investigation of transient effects.

\subsection{Procedure}

The experiments reported here are a straightforward extension of the drop deformation studies described by Bentley \& Leal (1986 b). With a drop maintained at the centre of the four-roll mill, the shear rate is increased by small increments of about $0.01 \mathrm{~s}^{-1}$ until a steady drop shape no longer exists. The corresponding shear rate is termed the critical shear rate $G_{c}$, or, in dimensionless terms, the critical capillary number $\mathbb{C}_{c}$. At this point the drop elongates continuously in the local flow field. When a certain elongation ratio $L / a$ is reached, the flow is stopped. Depending on the

† It may be noted that there is a small, but definite systematic increase in the data from still photographs relative to the digital-image data for low-viscosity-ratio drops. This difference is a consequence of a small time lag in manually triggering the still picture relative to the moment when a digital image is obtained. This time lag is accentuated for low-viscosity-ratio drops because they stretch more rapidly. 
viscosity ratio and the degree of elongation, the drop then either breaks up into a series of 'daughter' drops or else relaxes back to a spherical shape through a rather complex motion.

The objective of the experiments in which the flow is stopped is to approximate an abrupt step change in the suspending fluid from a steady motion to a state of rest. In order to achieve this, a necessary condition is that the characteristic timescale for the viscous response of the velocity field in the four-roll mill to changes in roller speed is small compared with any timescale of drop deformation. The present study was performed using Pale-4 Oil (and oxidized castor oil available from Cas Chem, Inc., Bayonne, N.J.) as the suspending fluid, with viscosity of approximately $50 \mathrm{P}$. The characteristic viscous response time for changes in the four-roll mill flow, $l_{\mathrm{c}}^{2} / \nu$, is thus $0.3 \mathrm{~s}$ (here $l_{\mathrm{c}}$ is the characteristic apparatus lengthscale, and $\nu$ is the kinematic viscosity of the suspending fluid). On the other hand, the characteristic timescale for interfacial-tension-driven changes in drop shape is $\mu a(1+\lambda) / \sigma$ and this is usually large compared with the viscous response time for the flow. In general, then, when the rollers are stopped, drop motion is driven strictly by interfacial tension and is a consequence of the non-equilibrium shape of the extended drop in an otherwise quiescent fluid.

All of the experiments were recorded on video tape for qualitative viewing and the results were quantified by analysing digital images of the deformed drop or by occasionally taking $35 \mathrm{~mm}$ still photographs. Experiments were performed for ten viscosity ratios between 0.01 and 12 and for five flow types $\alpha=1.0,0.8,0.6,0.4$ and 0.2. The drop fluids were a series of Dow Corning Silicone oils. The properties of these fluid systems were tabulated by Bentley \& Leal $(1986 b)$. The undeformed-drop radius varied between $0.05 \mathrm{~cm}$ and $0.1 \mathrm{~cm}$ and, in all cases, was small enough that the elongated drop remained in the central region of the device where the flow field is given approximately by (1). The Reynolds number $\rho G a^{2} / \mu$ was about $10^{-4}$ for these experiments.

\section{Results}

In this section we present the results of our experimental study of the elongation of liquid drops in two-dimensional strong flows, and the subsequent relaxation and/or breakup of the drops after the flow is stopped. First, we report qualitative observations of drop deformation and breakup, including the effects of varying both flow type and viscosity ratio. Secondly, we present quantitative results for the drop-elongation ratio $L / a$ as a function of time, as well as the critical elongation ratio necessary to ensure breakup for this special flow history. Our results will be compared with two existing theoretical analyses: (i) an extending, infinite fluid cylinder in an axisymmetric extensional flow and (ii) capillary-wave grow th on an infinite, stationary liquid cyclinder in a quiescent fluid. In the final section, we present a qualitative explanation for the dynamics of the observed relaxation and breakup phenomena and we describe briefly a new solution for the motion of an elongated drop in a quiescent fluid generated using the boundary-integral technique which supports this qualitative explanation.

\subsection{Qualitative behaviour}

We begin by describing the elongation and subsequent interfacial-tension-driven breakup in qualitative terms, using the results for the case $\lambda=2.4, \alpha=1.0$ (figure $6 a, b)$ to illustrate the phenomena. The first photograph in series $(a)$ is the undeformed 


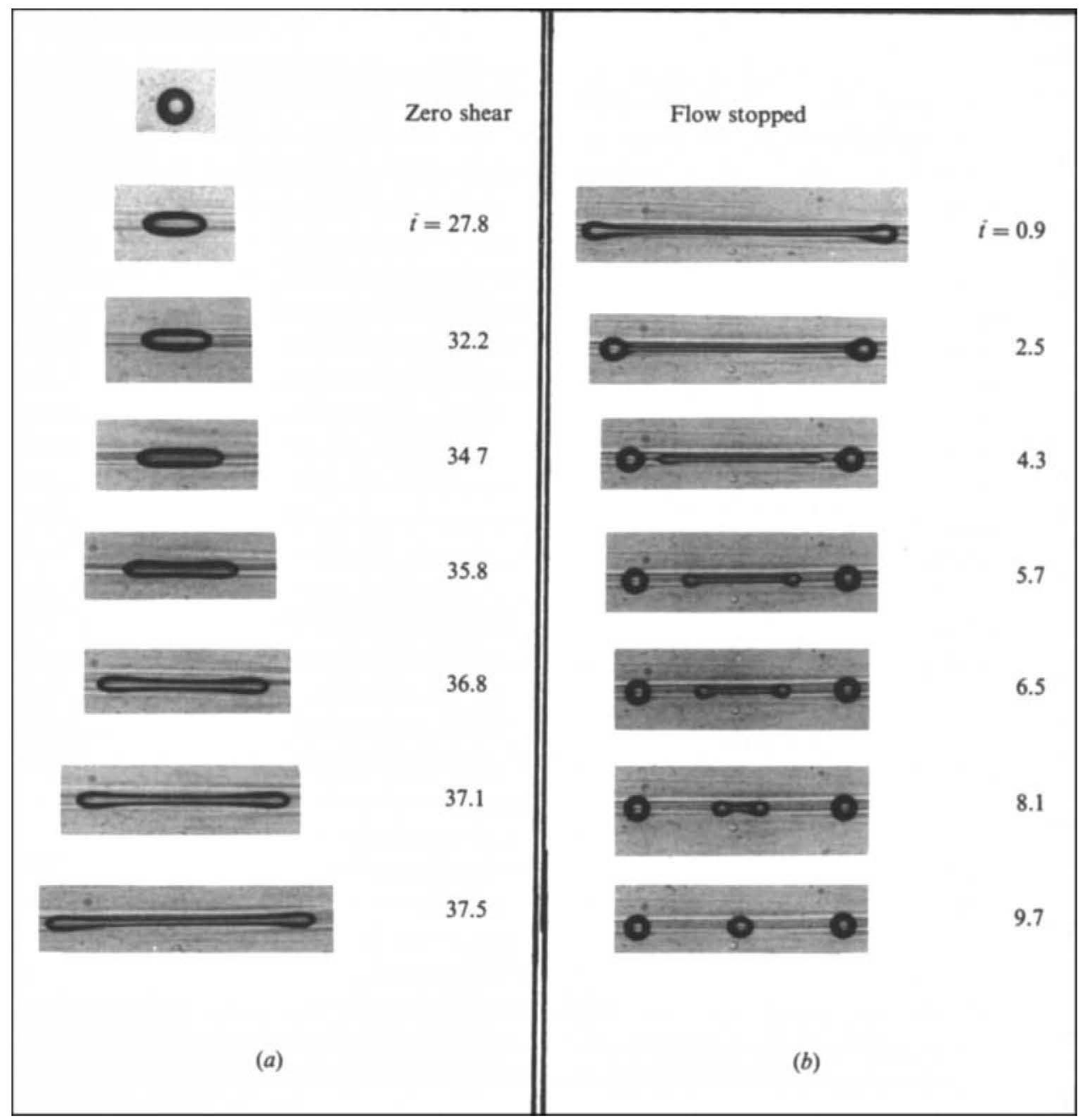

Figure 6. (a) Elongation of a liquid droplet, $\lambda=2.4, \alpha=1.0$, in a steady flow at the critical capillary number, $t=G_{\mathrm{c}} \alpha^{\frac{1}{2} t}, G_{\mathrm{e}}=0.132 \mathrm{~s}^{-1}$. (b) Relaxation and breakup after the flow is stopped abruptly 'end pinching'. The time is measured from the instant the flow ceases and $\bar{t}=G_{\mathrm{c}} \alpha^{\frac{1}{2} t}$.

drop, and the remaining photos exhibit the transient deformation of the drop while the flow is maintained at the critical capillary number, $\mathbb{C}_{c}=0.117$. The times listed to the right of the photographs (and all other photographs shown in this paper) are non-dimensionalized with respect to $G_{\mathrm{c}} \alpha^{\frac{1}{2}}\left(\bar{t}=G_{\mathrm{c}} \alpha^{\frac{1}{2}} t\right)$ and it is clear from the values listed that the initial elongation process is very slow. This is, in fact, true for all viscosity ratios, though the rate of elongation in this initial stage of transient deformation is observed to decrease as the viscosity ratio increases. During this slow initial elongation process, the sides of the drop are gradually flattened until the drop eventually develops a waist. Once the waist develops, the rate of drop elongation increases, as seen in the last three photos in series $(a)$, eventually approaching the rate of elongation of a fluid line element in the linear flow. During this period, the central section of the drop decreases rapidly in radius. Meanwhile, the ends retain their bulbous shape and appear to translate with only a small change in volume. 
While the flow is on, there is no evidence of capillary waves on the central cylindrical section. This was true for all the systems examined and will be discussed more fully in \$4.5. Furthermore, we found no case where the extending drop in a steady flow fractures at the middle while the flow was on, though it should be noted that the total elongation in our experiments was limited by the finite dimensions of the four-roll mill.

The sequence of photos, figure $6(b)$, shows the drop behaviour after the flow is stopped. The times shown are measured relative to the instant that the flow is turned off and, for consistency, have also been non-dimensionalized with respect to $G_{\mathrm{c}} \alpha^{\frac{1}{2}}$. The most interesting and important observation is that the breakup process, illustrated by the series of photos, is entirely different from the capillary-wave instability mechanism that is the basis for all existing theories of drop breakup! The motion leading to breakup is obviously driven by the finite interfacial tension of the interface. However, this motion is clearly a consequence of capillary-pressure variations near the ends of the drop rather than the instability of infinitesimal disturbances in the drop shape. We first notice that the ends immediately become almost spherical after the flow has stopped, forming a dumbbell-like shape, while the overall length decreases. The ends then proceed to pinch off, leaving a cylindrical thread of fluid which relaxes rapidly while the newly formed ends bulb up. However, in this particular case, the new ends do not pinch off and the central thread relaxes to form a single spherical drop. Just barely visible between each pair of drops is a tiny satellite drop. During the relaxation and breakup process exhibited in figure $6(b)$, capillary waves are never visible on the central, cylindrical section. Although this is not true in general, as we shall see in $\$ 4.5$, evidence of capillary-wave instability only appears for the most elongated drops that could be achieved in our four-roll mill while still maintaining the drop within the central region of homogeneous flow.

Qualitatively, the behaviour observed in figure $6(b)$ is typical of all fluid systems studied. We call the new breakup mechanism 'end pinching'. It appears that the final drop size distribution obtained via the 'end-pinching' mechanism is determined by the rate at which the ends bulb up and contract toward the drop centre, relative to the rate at which the ends pinch off. Evidently, the interfacial-tension-induced flow responsible for 'end pinching' occurs on a much shorter timescale than the growth of capillary waves, at least for the particular case shown in figure 6. Theories of drop breakup and resulting predictions of drop size distributions based upon a capillarywave instability mechanism will clearly not suffice in such circumstances.

\subsection{Effect of flow type}

Before considering the effect of varying the viscosity ratio on the dynamics of the elongation and 'end-pinching' processes, it is worthwhile to examine the effect of flow type. Bentley \& Leal (1986b) have shown that a drop that passes through a series of quasi-steady states becomes oriented with its long axis along the exit streamline of the flow (figure 1) as the critical capillary number is approached. At this orientation, the effective strain rate of the fluid on the drop (i.e. the strain rate along its axis) is $G_{c} \alpha^{\frac{1}{2}}$.

Now, figure 7 illustrates the elongation and breakup process for $\lambda=0.09$ and two different flows, $\alpha=0.6$ and 0.2 respectively. The main difference, other than flow type, is that the critical shear rate $G_{\mathrm{c}}$ is approximately 1.5 times larger for the $\alpha=0.2$ flow. Otherwise, the similarities are rather remarkable. As before, the drop undergoes a very slow initial elongation followed by a period of rapid extension prior to stopping the flow. Notice that the $\alpha=0.6$ drop was allowed to stretch further before the flow 


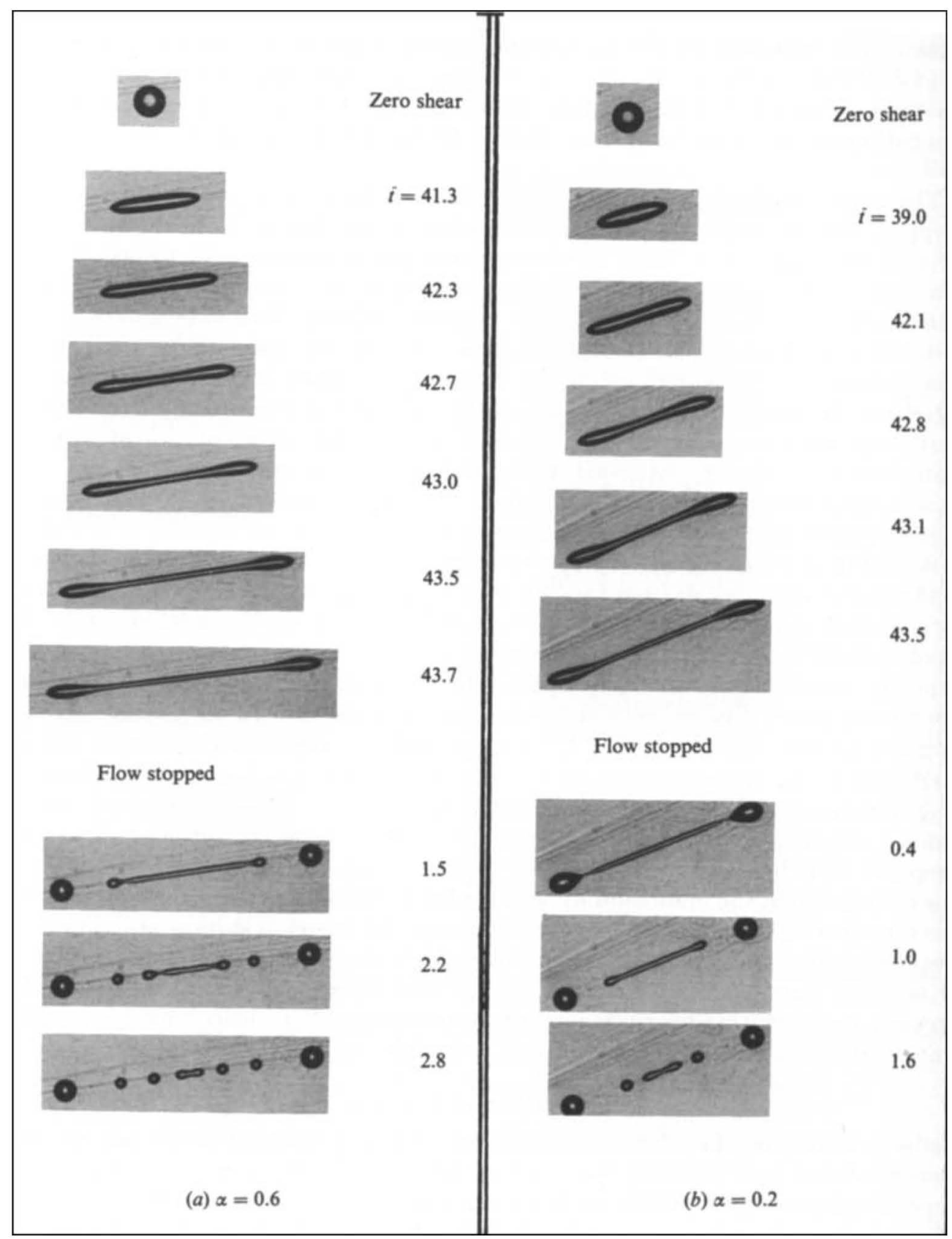

Figure 7. The effect of flow type on the elongation process and on the subsequent relaxation and breakup phenomena. Viscosity ratio is held constant, $\lambda=0.09,(a) \alpha=0.6, G_{\mathrm{c}}=0.224 \mathrm{~s}^{-1} ;(b)$ $\alpha=0.2, G_{\mathrm{c}}=0.33 \mathrm{~s}^{-1}$.

was stopped, which leads to more drop fragments being formed. The dynamics of the breakup process, though, are very similar for the two cases in spite of the fact that the final extended shapes are somewhat different. This suggests that drop breakup in a quiescent fluid depends primarily on the global geometry (rather than on any local feature of the shape) and the basic shape of the elongated drops generated by 


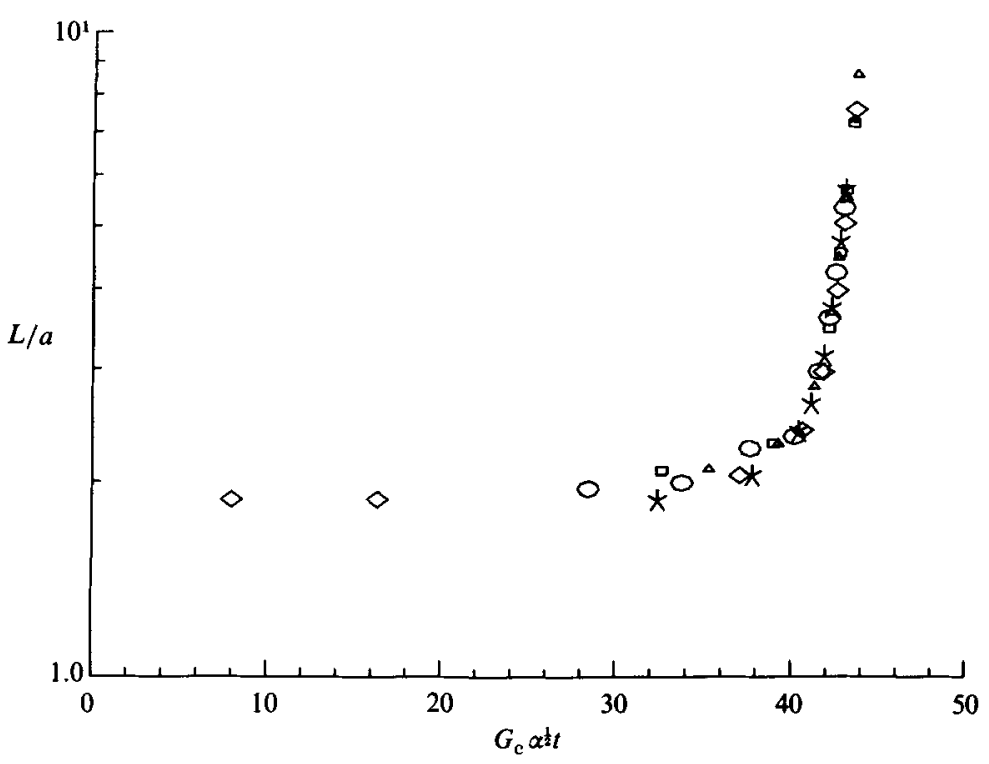

Figure 8. The effect of flow type on the elongation process - elongation ratio $L / a$ as a function of dimensionless time $G_{c} \alpha^{\frac{1}{2} t}$. This figure illustrates that the effect of flow type is only to modify the timescale of the elongation process. The data for each experiment ends when the flow is stopped: $\lambda=0.09 ; \oslash, \alpha=1.0 ; \star, 0.8 ; \triangle, 0.6 ; \diamond, 0.4 ; \square, 0.2$.

these flows is very similar. The 'end-pinching' mechanism is observed and, in these cases, the process repeats itself on the middle thread. Since the shape of this thread, immediately after its formation, is different from the shape of the originally stretched drop, we again see that it is the overall elongated shape and not local details that dominates and is responsible for the 'end-pinching' breakup process.

Figure 8 illustrates the rate at which the elongation ratio $L / a$ varies with dimensionless time $G_{\mathrm{c}} \alpha^{\frac{1}{2}} t$ for the drops shown in figure 7. Additional data are also presented for the same $\lambda$ but different $\alpha$. Here we focus attention on the rate of elongation as a function of flow type, for constant $\lambda$. Hence, the data for each experiment ends when the flow is stopped. Because $G_{\mathrm{c}} \alpha^{\frac{1}{2}}$ is the effective extension rate along the exit streamline where the elongated drops align, it is a reasonable choice for scaling the time while the flow is on, and this is indicated by the agreement in the data of figure 8 for the various values of $\alpha$.

It should be noted that the very slow elongation process that characterizes the initial stage of transient deformation means that a small error in experimentally determining the critical capillary number makes a large difference in the origin of the time axis for each experiment. Consequently, all the plots of $L / a$ versus time in this paper have been adjusted so that the steeply sloping portions of the curves overlap. This adjustment affects the relative position of the curves, but has no effect on their shape. From figure 8 it is clear that the effect of flow type $\alpha$ is only to modify the timescale for elongation of the drop, at least for the particular flow history that we examine in this paper. Hence, the photographs in figure 7 and the rate-of-elongation data presented in figure 8 demonstrate that the qualitative nature of the transient deformation and relaxation processes are essentially independent of flow type for a given value of $G_{c} \alpha^{\frac{1}{2}}$. 


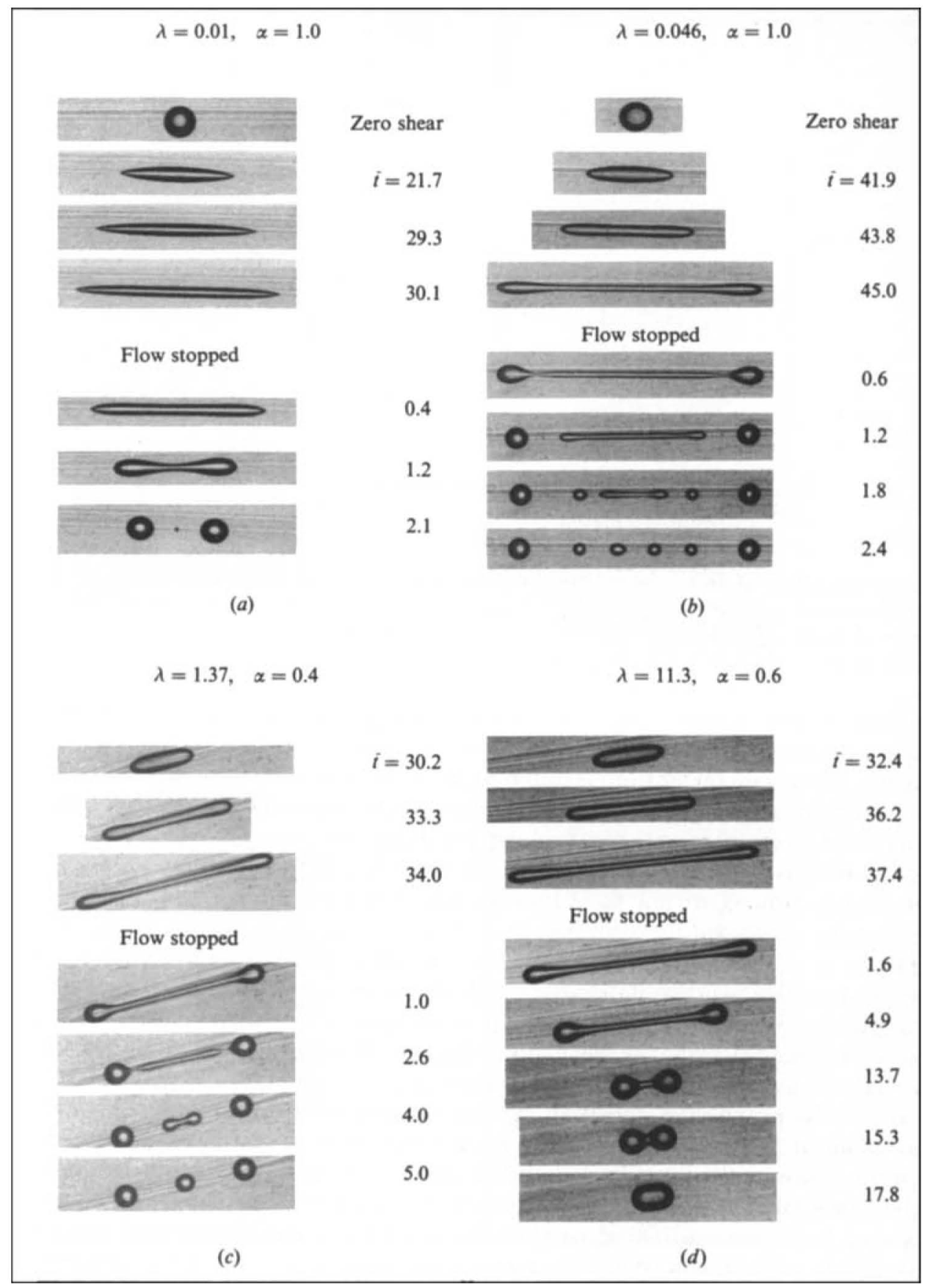

Figure 9. The effect of viscosity ratio on the elongation and breakup phenomena. $(a) G_{\mathrm{c}}=0.260 \mathrm{~s}^{-1}$, $\mathbb{C}_{\mathrm{c}}=0.26$. (b) $G_{\mathrm{c}}=0.235 \mathrm{~s}^{-1}, \mathbb{C}_{\mathrm{c}}=0.21$. (c) $G_{\mathrm{e}}=0.187 \mathrm{~s}^{-1}, \mathbb{C}_{\mathrm{c}}=0.18$. (d) $G_{\mathrm{c}}=0.135 \mathrm{~s}^{-1}, \mathbb{C}_{\mathrm{c}}=0.14$

\subsection{Effect of viscosity ratio}

Figure 9 illustrates the elongation and breakup process for the entire range of viscosity ratios that we studied. Although the flow type is different for the various cases shown in figure 9 , we have already demonstrated that $\alpha$ has no effect other than 


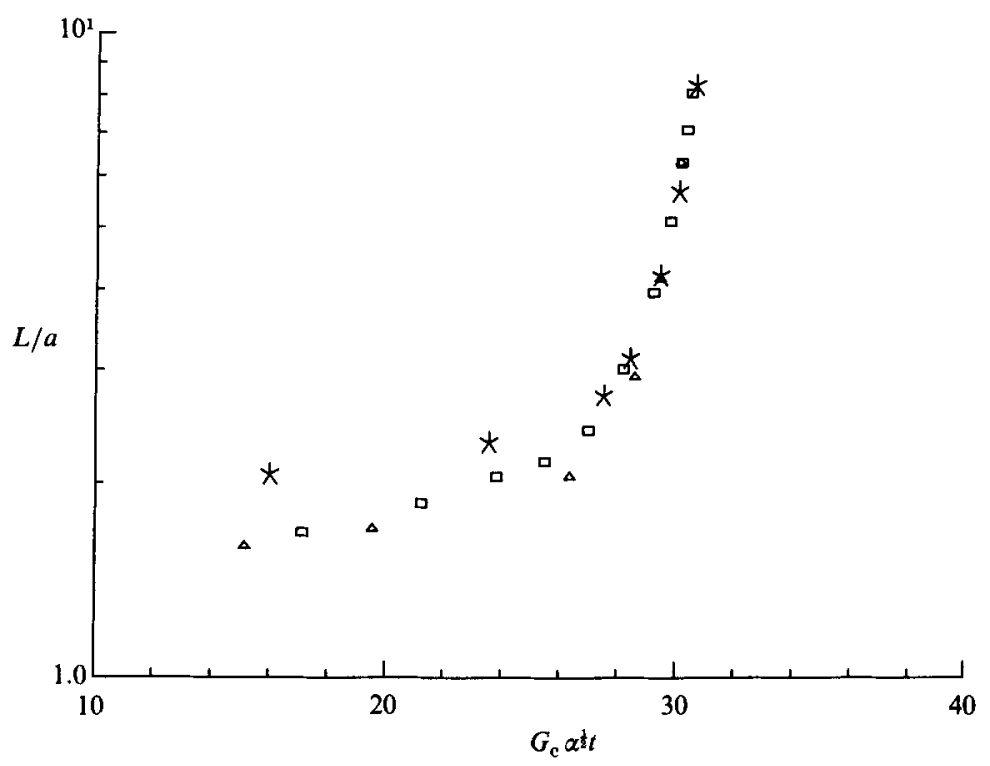

Figure 10. Elongation ratio $L / a$ as a function of dimensionless time for the experiments shown in figure $9(b, c, d)$. The data indicate the remarkable similarity in stretching rate for $(L / a) \gtrsim 3$ in spite of the 300 -fold difference in viscosity ratio. $\star, \lambda=0.046, \alpha=1.0 ; \triangle, \lambda=1.37, \alpha=0.4$; $\square, \lambda=11.3, \alpha=0.6$.

determining the critical shear rate and hence the timescale for elongation. The differences apparent among the experiments shown in figure 9 are a consequence of changes in the viscosity ratio $\lambda$.

Figure $9(a)$ shows a sequence of photographs illustrating the transient behaviour of a drop with viscosity ratio 0.01 . In low-viscosity-ratio experiments such as this one, the drop attains highly elongated steady shapes prior to achieving the critical shear rate where continuous elongation occurs, and the ends of the drop are much more pointed than we saw in either figures 6 or 7 . During elongation with the flow on, the shape of the drop does not change dramatically. Except for a small region near the ends which remains almost pointed, the width of the elongating drop is nearly constant over its entire length at any instant. When the flow is stopped, however, the ends rapidly become rounded, the overall length is reduced significantly, fluid drains from the centre and the drop breaks. Two daughter droplets are formed in the case illustrated here with a barely visible satellite drop between them.

Two intermediate viscosity ratio cases $\lambda=0.046$ and 1.37 are shown in figure $9(b, c)$ respectively, and the qualitative behaviour is very similar to the cases $\lambda=1.4$ and 0.09 examined in figures 6 and 7 respectively. In the present figures, the initial onset of stretching of the drop occurs at moderate deformations. In other words, the maximum steady deformation is relatively small in these cases, as already shown by Bentley \& Leal $(\mathbf{1 9 8 6} b)$. For both experiments, breakup is evidently a consequence of the 'end-pinching' mechanism.

Figure $9(d)$ illustrates the drop behaviour for the highest viscosity ratio examined in this study, $\lambda=11.3$. The new qualitative, dynamical feature we observe is that the drop relaxes back to a spherical shape after the flow is stopped, even though it is highly elongated and the ends bulb up to produce a dumbbell-like shape. No pinching-off of the ends occurs. Instead, the ends are pulled toward the drop centre and engulf the cylindrical portion of the drop as they move. It is interesting that the diameter of the central portion of the drop remains essentially contant until the 
separation between the drops ends is approximately equal to the end diameter. Again, capillary waves are not observed.

Examination of the photographs in figure $9(b-d)$ illustrates that, despite the 300 -fold difference in viscosity ratio, the shapes of the intermediate and highviscosity-ratio drops are remarkably similar while the drop is elongating in a steady flow at the critical capillary number. Furthermore, the rates of stretching of these different viscosity drops, following the slow initial deformation, are also remarkably similar. This is clearly evident in figure 10 which presents the elongation ratio $L / a$ as a function of dimensionless time $G_{\mathrm{c}} \alpha^{\frac{1}{2} t}$ for the experiments shown in figure $9(b-d)$. Data are only presented for the period while the flow is on, the results having been adjusted so that the steeply sloping portions of the curves overlap. As is evident in the photographs, however, the similarities in behaviour end after the flow stops. Not only does the high-viscosity-ratio drop not fragment, in spite of having a comparable initial $L / a$ and shape, as the intermediate viscosity ratio drops but, as is to be expected, the time period over which relaxation occurs is much longer the larger the viscosity ratio (when examining the relaxation process it is necessary to remember that the time has been scaled with respect to $G_{\mathrm{c}} \alpha^{\frac{1}{2}}$ and, for $\lambda \lesssim 10, G_{\mathrm{c}}$ decreases with increasing $\lambda$ ). Because the degree of elongation in the three cases is similar, the differences observed for the high- $\lambda$ case are evidently due to the effects of viscosity ratio.

With reference to the lowest viscosity ratio, $\lambda=0.01$, if the drop is allowed to stretch further than shown in figure $9(a)$, we find that it also stretches at the same rate as the higher-viscosity-ratio drops shown in figure 10 . However, the dynamics following cessation of the flow appear qualitatively different from the intermediateand high- $\lambda$ experiments owing both to the lower viscosity ratio and the initial, pointed shape (which, of course, is also a consequence of the lower viscosity ratio). A qualitative explanation for the effect of viscosity ratio on the elongation/breakup process, as exhibited by these photographs, will be presented in $\$ 5$.

\subsection{Effect of $L / a$; critical elongation necessary to ensure breakup once the flow is stopped}

We have yet to address specifically the question of how the initial degree of elongation affects the relaxation and breakup dynamics. First, we must reiterate that in no case did we observe breakup while the flow was on. Drop breakup, and, consequently, the final drop size distribution for a given viscosity ratio, were dependent on the elongation ratio $L / a$ prior to stopping the flow.

It should not be surprising, in the light of the photographs presented in this paper, that drops that were extended only a little past their steady shape relaxed back to a sphere without fragmenting over the entire range of viscosity ratios studied. In addition, in all cases there existed a critical elongation ratio above which the drop fragmented when the flow ceased.

For each of the viscosity ratios studied, several experiments were performed to determine the critical elongation ratio for drop breakup. One way to illustrate the results of varying the elongation ratio is to examine data for $L / a$ as a function of dimensionless time. Such data are shown in figure $11(a-c)$ for $\lambda=0.018,0.47$ and 5.7 respectively, and for a range of final elongation ratios at the point when the flow is stopped. These plots are very similar to figures 8 and 10 . Here, however, we systematically vary $L / a$, holding $\lambda$ constant, and observe the effect on the relaxation/breakup process and subsequent drop size distribution. For each experiment, we mark by a horizontal arrow, the point where the flow is stopped. Measurements of 
Transient effects in drop breakup
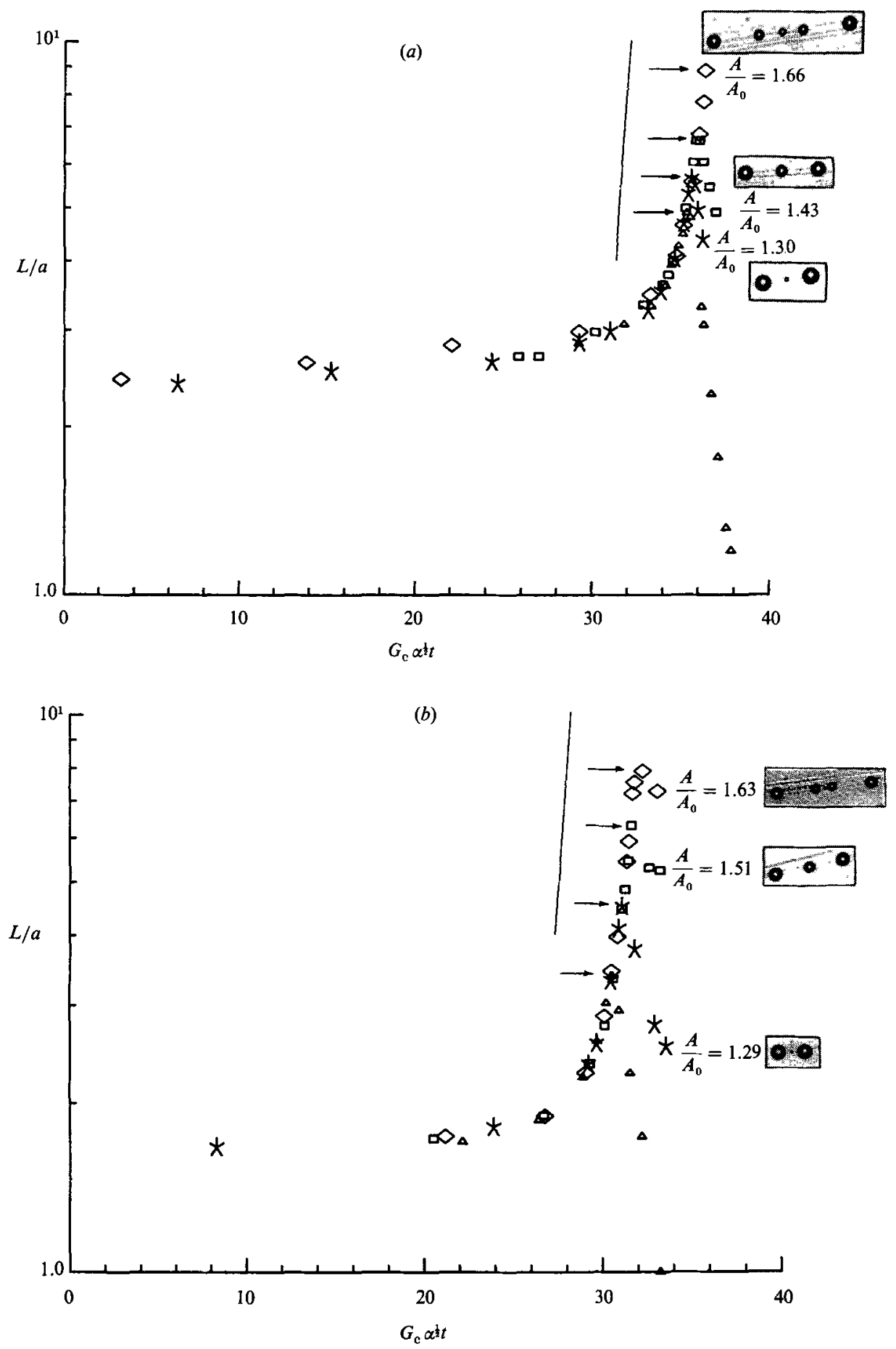

Figure $11(a, b)$. For caption see page 148. 


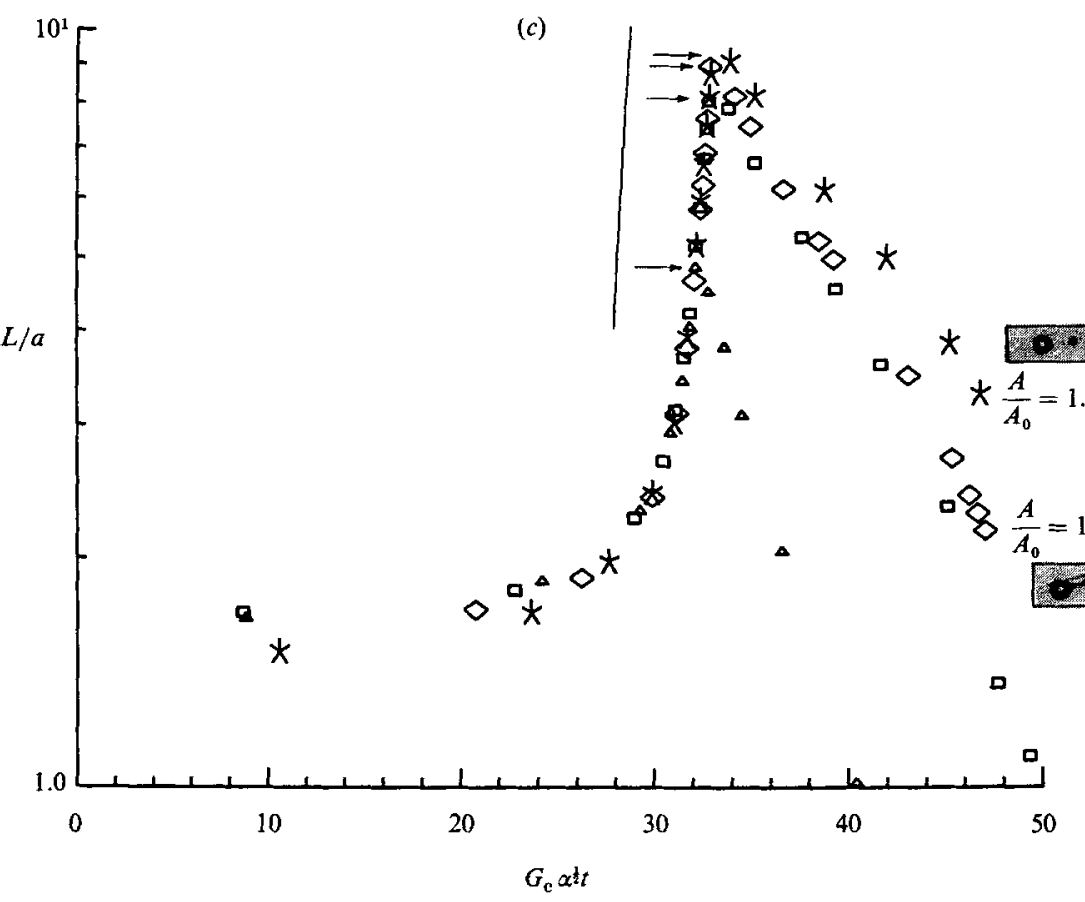

Figure 11. The effect of $L / a$ on the relaxation and breakup process. $(a) \lambda=0.018,(b) 0.47,(c) 5.7$. The horizontal arrows indicate when the flow is stopped. Data points for each experiment then continue until the first fragment separates from the main drop. $A / A_{0}$ denotes the fractional increase in interfacial area due to the fragmentation. The solid, almost vertical line indicates the rate at which a fluid element stretches in the linear flow. $\triangle, \alpha=1.0 ; \square, 0.8 ; \star, 0.6 ; \diamond, 0.6$.

$L / a$ then are continued until the first fragment separates from the main drop. The value of $L / a$ at this instant is indicated by the last data point for each symbol. Although motion is driven by interfacial tension after the flow is stopped so that a more appropriate time scale is $\mu a(1+\lambda) / \sigma$, for convenience, the timescale was not changed in this portion of the plot. In each figure, data that returns to $L / a=1$ indicates that the drop relaxes back to a sphere without fragmenting. When breakup does occur, we also include a photograph of the final dispersed state of the drop after breakup and indicate the fractional increase in interfacial area, denoted by $A / A_{0}$, that is generated by the fragmentation process (the bottom photograph in figure $11 c$ shows the drop shortly before it fragments into two equally sized droplets). These figures reiterate some features that were exposed earlier by the photographs, namely that provided $L / a$ is not too large, a considerable shortening of drop length occurs prior to fragmentation, and the relaxation is slower for higher viscosity ratios. Finally, the solid, almost vertical line in each figure illustrates the expected slope if the drop were to stretch at the same rate as a fluid element in the linear flow. It is clear that as the drop becomes increasingly elongated this asymptotic behaviour is approached.

These figures illustrate that for small enough $L / a$ the drop relaxes back to a sphere. However, above a critical elongation ratio the drop breaks and the number of drop fragments (and hence interfacial area) increases with increasing $L / a$ beyond this critical value.

For all viscosity ratios, the critical elongation ratio necessary to guarantee drop 


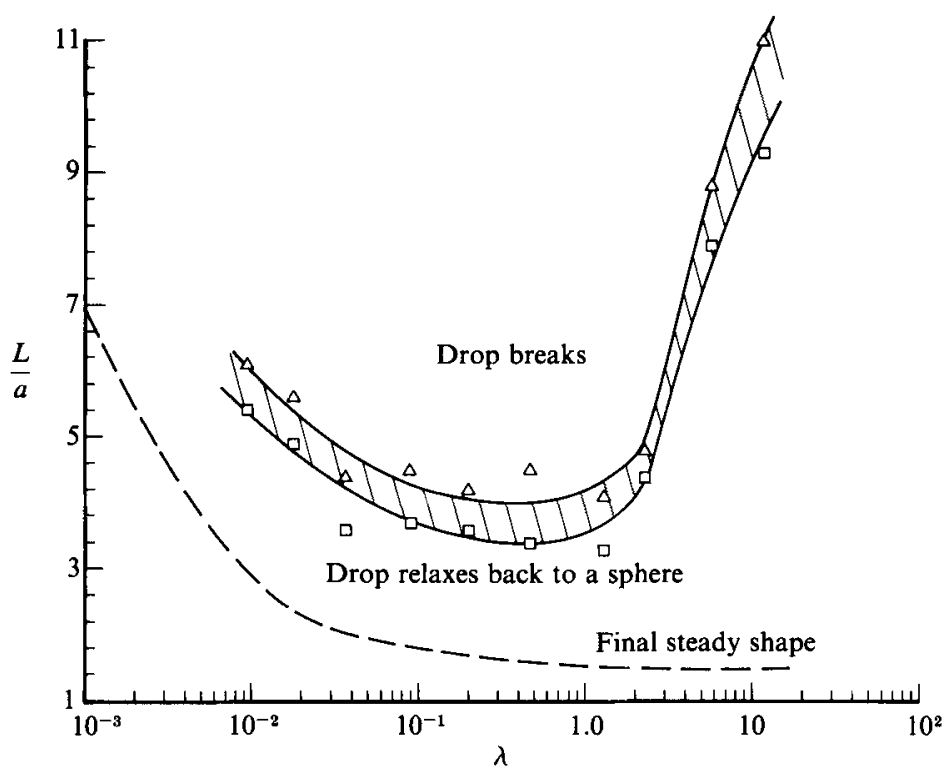

Figure 12. Critical elongation ratio necessary to ensure breakup, following an abrupt halt of the flow, as a function of viscosity ratio. Triangles denote the smallest $L / a$ for which a drop was observed to breakup. Squares denote the largest $L / a$ for which a drop relaxed back to a sphere. The shaded region denotes the uncertainty in the critical elongation ratio. The dashed line indicates the $L / a$ values of the final steady shapes.

breakup for the flow history that we examined is summarized in figure 12. The squares denote the largest elongation ratio for which the drop relaxed back to a sphere and the triangles denote the smallest elongation ratio for which breakup was observed upon cessation of the flow. The cross-hatched region therefore denotes the uncertainty in the magnitude of the critical elongation ratio. The dotted line in this figure represents $L / a$ for the most deformed steady shapes that were observed experimentally by Bentley \& Leal (1986 b). Examination of the unsteady elongation and breakup for $\lambda \approx 10^{-3}$ has been very difficult because the steady shapes are very long and slender. The data in figure 12 illustrate, as found by Grace (1971), that the critical elongation necessary to ensure breakup is large compared with the maximum stable shape.

Figure 12 also clearly illustrates a difficulty in breaking drops with either a high or low viscosity ratio. As shown by the photographs in figure 9 , high- $\lambda$ drops are able to relax back to a spherical shape before either capillary-wave instabilities on the central cylindrical portion or the dynamics of 'end pinching' cause fragmentation. On the other hand, low-viscosity-ratio drops are difficult to break primarily because a high degree of elongation (and a high capillary number) is necessary before the drop even begins to elongate with time in a steady flow.

The graph exhibits a minimum in the critical $L / a$ in the range $0.1 \lesssim \lambda \lesssim 2.0$ and this is qualitatively similar to results reported by Grace (1971) for hyperbolic flow. A quantitative comparison is difficult, however, because Grace reported all results in terms of $D$, which is very insensitive to increased extension for the highly elongated drops formed in these experiments. As discussed above, our results hold for all $\alpha$. 


\subsection{Capillary waves}

In $\$ \S 4.1,4.3$ and 4.4 we have noted that capillary-wave instability is not observed during the elongation of drops in the present experiments. Furthermore, there is at least a range of elongation ratios where the drop is highly elongated but the breakup mode, in the absence of any external flow, is due to 'end pinch-off' and not due to the growth of capillary waves. Both of these results are surprising in view of the previous work on the drop breakup problem and deserve further discussion and investigation.

First, we consider the fact that no capillary waves are observed during the elongation of the drop, even though the midsection of the drop becomes long and cylindrical and $L / a$ values as large $O(15)$ are attained. Mikami et al. (1975) published a theoretical stability analysis for an infinite fluid cylinder in a second fluid that is undergoing an axisymmetric extension. The theory shows that the fluid cylinder behaves exactly as a fluid element in this flow and we have already seen that this asymptotic behaviour is approached as the drop elongates during the experiment. In general, though, the theoretical results are quite complicated with the net effect being that the elongation of the thread decreases the overall growth rate of capillary waves. Nevertheless, some disturbances are still predicted to grow exponentially and this suggests that breakup is possible via capillary-wave growth even when the drop is extending. In addition, Mikami et al. (1975) demonstrate that the predictions of the linear stability analysis are valid for the two-dimensional extensional flow generated in the four-roll mill. Indeed, these investigators report experimental observations that qualitatively support their analysis and show breakup of a fluid thread in a steady two-dimensional hyperbolic flow. The main difference between their experiment and ours is that they achieved much greater elongation ratios than the maximum values of $O(15)$ that we obtained in our experiments. Presumably, a drop must become much more elongated before capillary-wave instabilities become evident during its extension, but this could not be tested in our apparatus because of constraints imposed by the size of the linear flow region and by resolution limits of the video camera in the control system.

When the flow is turned off after the drop has been highly extended, the midsection would appear, even with the ends being shed via 'end pinching' to approximate a stationary liquid cylinder. The stability of an infinite, stationary fluid cylinder in a second viscous fluid was studied by Tomotika (1935), Rumscheidt \& Mason (1961), Lee \& Flumerfelt (1981) and Lee et al. (1981). As demonstrated by Tomotika, although an infinite fluid cylinder suspended in a quiescent fluid is a perfectly valid solution to the governing equations, $\boldsymbol{u}=0$ everywhere, it is unstable with respect to small disturbances with wavelength greater than the cylinder circumference. Crudely, then, capillary-wave instability cannot occur on elongated drops unless the length is greater than the circumference. However, for longer drops, capillary-wave growth should be expected. The fact that we see no evidence of capillary waves in the majority of our experiments, even when the initial length of the extended drop is several times its circumference, is probably because the 'end-pinching' process occurs on a short timescale relative to the time required for small capillary-wave disturbances to achieve finite amplitude. Thus, the drop is reduced to a length that can no longer support capillary-wave growth before significant growth of the initial disturbances can occur. However, this 'explanation' suggests that capillary waves should eventually become evident if we simply make the drop long enough - for such a long drop, breakup will occur initially via the 'end-pinching' mechanism, but now 


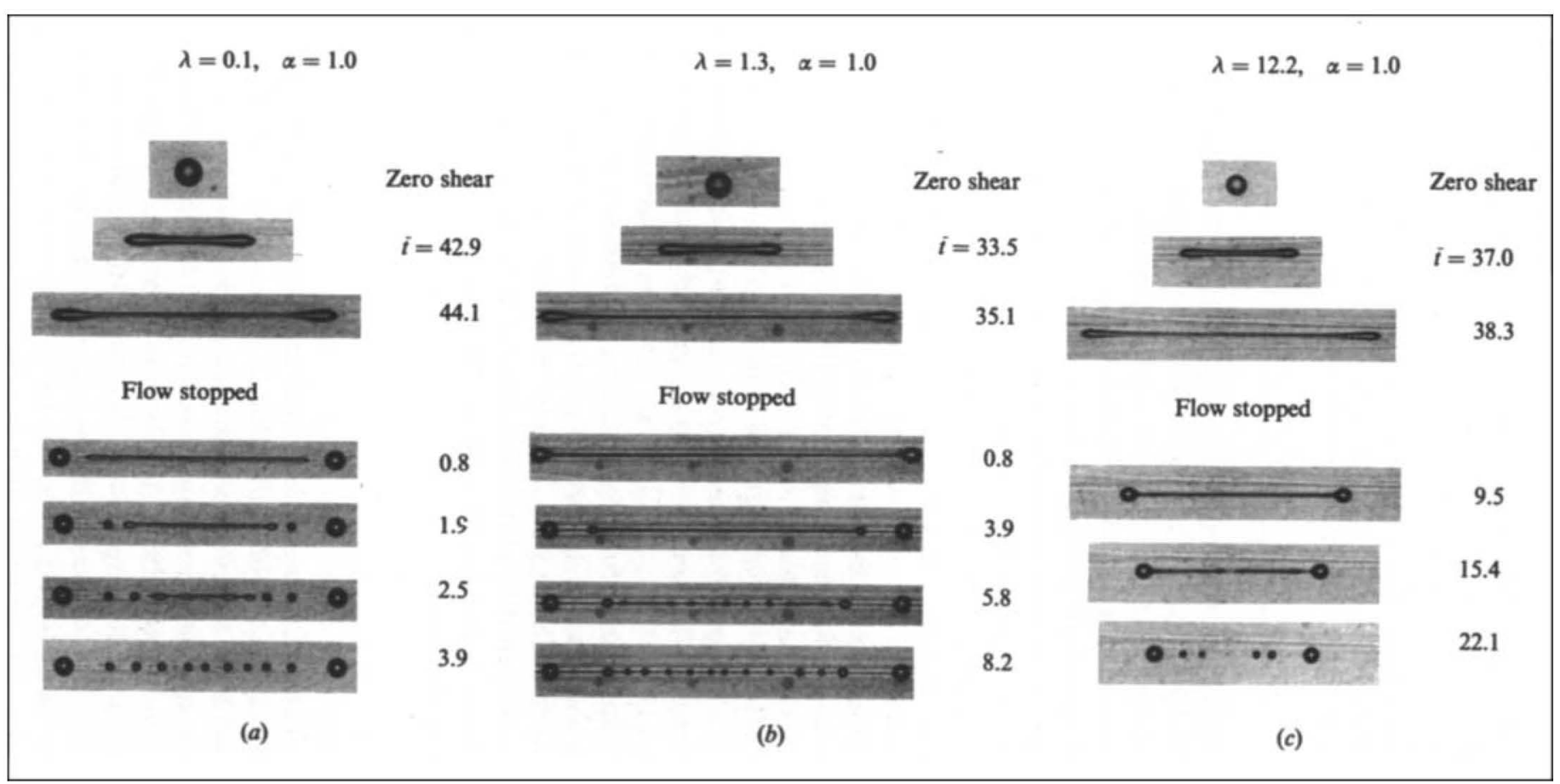

Figure 13. Development of capillary-wave instabilities. Notice that capillary waves are not visible while the drop stretches (a) $G_{\mathrm{c}}=0.306 \mathrm{~s}^{-1}, \mathbb{C}_{\mathrm{c}}=0.18 .(b) G_{\mathrm{c}}=0.218 \mathrm{~s}^{-1}, \mathbb{C}_{\mathrm{c}}=0.12$. (c) $G_{\mathrm{c}}=0.234 \mathrm{~s}^{-1}, \mathbb{C}_{\mathrm{c}}=0.10$. 


\begin{tabular}{clll} 
& \multicolumn{2}{c}{$\left(\frac{2 \Pi R_{0}}{A}\right)_{\text {critical }}$} & \\
$\lambda$ & 0.1 & 1.3 & 12.2 \\
theory & 0.57 & 0.55 & 0.39 \\
exp. & 0.58 & 0.57 & 0.50
\end{tabular}

TABLE 1. Comparison of experiment and theory for the instability of infinite, stationary liquid cylinders. $A$ denotes the disturbance wavelength and $R_{0}$ the thread radius. The theoretical predictions are obtained in the limit of negligible inertial effects, $\rho \sigma a / \mu^{2} \ll 1, \hat{\rho} \sigma a / \hat{\mu}^{2} \ll 1$ (Lee \& Flumerfelt 1981).

capillary waves may appear on the remaining elongated thread simply because the drop was so long initially that the timescale for its complete breakup via 'end pinching' exceeds the timescale for growth of capillary waves.

Based upon these ideas, we set out to search for evidence of capillary-wave growth by simply producing increasingly elongated drops. The results of this search confirm our qualitative explanation. Several examples of cases that show significant capillarywave growth are shown in figure 13 . In these cases, the presence of capillary-wave instability produces a striking and abrupt transition in the mode of breakup in the middle of the breakup process. At first, the drop begins to breakup via the 'end-pinching' mechanism which we identified earlier in this paper. Then, however, there is an abrupt appearance of finite-amplitude waves on the remaining thread, which leads to simultaneous breakup into a line of small drops. The fact that this latter process is due to capillary-wave instability is confirmed by the comparison, shown in table 1, between the wavelength $(A)$ obtained from the experiments and the wavelength of the fastest-growing linear mode calculated from the linear stability theory in the limit of negligible inertial effects, $\rho \sigma R_{0} / \mu^{2}, \hat{\rho} \sigma R_{0} / \hat{\mu}^{2} \ll 1$, where $R_{0}$ is the thread radius. Experimentally, $\rho \sigma R_{0} / \mu^{2} \approx 10^{-4}$. The agreement is excellent for $\lambda=0.1$ and 1.3. The discrepancy for $\lambda=12.2$ may be because there is a relatively strong flow induced in the fluid by the initial contraction in length which occurs to a much greater extent for drops with a large viscosity ratio. Of course in the capillary-wave theory, an infinite, stationary thread is assumed and it is perhaps surprising that there is good agreement between the theory and experimental data for an elongated drop in any case.

\section{Discussion}

Of all the experimental observations described in the preceding section, the most important, and interesting, is the identification of the mode of interfacial-tensiondriven breakup that we have termed 'end pinching'. Qualitatively, it is clear that the mechanism is a consequence of motion generated via capillary pressure gradients in the region near the end of the drop. An obvious question, though, is why drops of intermediate viscosity ratio break via this mechanism when they are only modestly extended, while more-viscous drops do not break until $L / a$ is considerably larger. From our observations, it can be seen that a general precursor to 'pinch-off' is the development of a local 'waist' just inside the bulbous end. Once such a local minimum in the radius occurs, there will be an associated local pressure maximum inside the drop due to capillary forces and an obvious mechanism for increase in the depth of the minimum, subsequently leading to pinch-off. However, this simple observation does not explain why the local minimum in radius occurs in the first place, nor why it occurs less readily for drops of large viscosity ratio compared with 
(a)
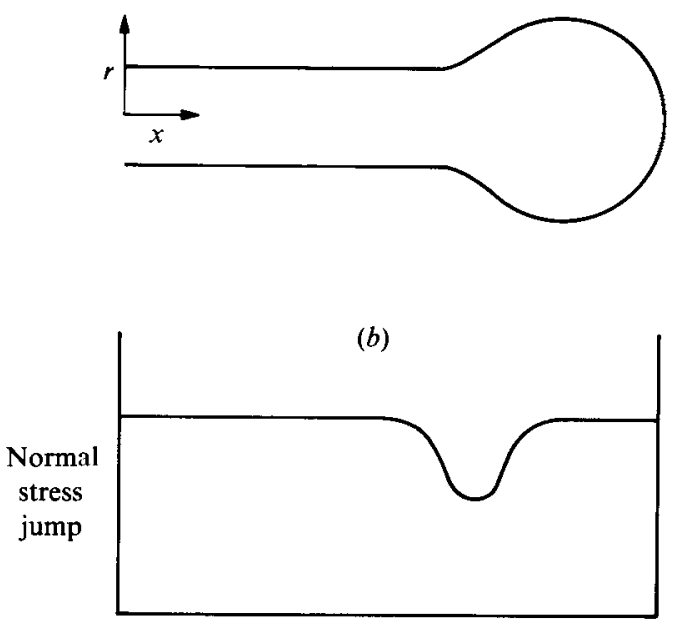

Distance along drop axis

Figure 14. (a) Typical drop shape shortly after the flow is stopped. Radius of bulbous end is twice the radius of the cylindrical midsection. (b) Approximate normal stress jump across the interface.

$\lambda=O(1)$. This latter question is particularly perplexing in view of the similar drop shapes in the initial stages of the relaxation/breakup process.

\subsection{A qualitative description of 'end pinching'}

A starting point in attempting to understand 'pinch-off' is the recognition, from dimensional analysis, that the qualitative features of the interfacial-tension-driven flow are determined completely by the viscosity ratio and the initial shape of the drop, including the value of $L / a$. One might, at first, suppose that the differences between the relaxation/breakup process for drops of intermediate and large viscosity ratio are a result of some viscosity-ratio-dependent detail of the drop shape at the instant when the external flow is stopped. However, this appears unlikely for several reasons. First, when 'end pinching' occurs, it does so for any elongation ratio greater than some minimum value even though the initial drop shape (as described, say, by the ratio of the radius of the bulbous end to the radius of the cylindrical midsection) varies with $L / a$. Secondly, the initial shapes for small and intermediate $\lambda$ are markedly different in the region near the end of the drop and yet 'end pinching' occurs in both cases. Finally, when 'end pinching' initially takes place, it often leaves an elongated central fragment which develops bulbous ends and may pinch-off in the same manner in spite of the fact that its shape is clearly different from the original extended shape. Hence, it would appear that the mechanism of 'end pinching' depends on the global geometry of the drop (and viscosity ratio), rather than on any local details of the shape. This was indicated previously in the discussion of figure 7 .

A more-plausible suggestion is that the difference between moderate- and highviscosity-ratio systems is due to differences in the relative rates of the flow in different regions of the drop which arise from the relative viscosities of the drop and continuous phases. In order to develop this concept, it is necessary to begin with a general description of the relaxation process. Let us begin by considering the internal pressure distribution associated with capillary forces for an interface of the shape shown in figure 14(a), which is qualitatively typical of the observed drop shapes in all cases soon after the flow is stopped. For illustration purposes, we have chosen the 
radius of the bulbous end to be twice the radius of the cylindrical midsection. Let us denote the stress tensor as $\boldsymbol{T}$ and the unit normal from the droplet phase to the continuous phase as $\boldsymbol{n}$. Then, if the drop surface is described in cylindrical coordinates as $r=f(x)$, the normal stress jump is given by

$$
(\boldsymbol{T} \cdot \boldsymbol{n}-\hat{\boldsymbol{T}} \cdot \boldsymbol{n}) \cdot \boldsymbol{n}=\frac{1}{R_{1}}+\frac{1}{R_{2}}=\frac{1}{\left[1+f^{\prime 2}\right]^{\frac{1}{2}}}\left[\frac{1}{f}-\frac{f^{\prime \prime}}{1+f^{\prime 2}}\right]
$$

where $R_{1}$ and $R_{2}$ are the principal radii of curvature of the surface, and $f^{\prime}$ and $f^{\prime \prime}$ are the derivatives of the shape function $f$, with respect to the axial coordinate $x$. The normal stress jump corresponding to the shape plotted in figure $14(a)$ is depicted qualitatively in figure $14(b)$. Now, let us suppose that the shape is fixed, so that the normal stress jump can be interpreted in terms of capillary-pressure variations within the drop. Then, we see that the pressure is highest near the end of the drop where the radius of curvature is concave in planes both parallel and perpendicular to the drop axis, goes through a minimum with decreasing $x$ because the drop surface in the plane parallel to the drop axis becomes convex, and finally increases to a constant value in the central portion of the drop.

This capillary pressure gradient will tend to induce flow both from the end of the drop and from the central cylindrical region. However, the flow from the end of the drop can occur without large velocity gradients in the internal fluid, since the drop end can translate without a significant change in shape. Thus, this motion is resisted mainly by viscous effects in the outer fluid. Motion of the bulbous end towards the pressure minimum causes the convex region (and hence the pressure minimum) to move toward the drop centre as well, so that the driving force for continued end movement is maintained. On the other hand, the dynamics of the flow from the central portion of the drop towards the pressure minimum is qualitatively different, because this flow requires significant velocity gradients within the inner fluid (note that the fluid is stagnant at the drop centre). As a consequence, this motion is inhibited primarily by the droplet viscosity. It will be noted, however, that a local 'neck' in the shape will tend to form as a consequence of a flux of fluid from the cylindrical region towards the pressure minimum.

Given this qualitative picture of interfacial-tension-driven changes in the drop shape, the observed differences between high-and low-viscosity-ratio systems may be 'explained' in the following manner. In high-viscosity-ratio systems, the ends are drawn toward the middle as described above, with the rate of this process controlled mainly by the relatively low-viscosity outer fluid. Drainage from the central portion, on the other hand, is comparatively slow since it is controlled by the higher-viscosity droplet fluid. As a result, movement of the pressure minimum and of the bulbous ends occurs sufficiently fast relative to the flow from the central region that a significant 'neck' in the shape cannot form unless the drop is highly elongated. In contrast, in lower-viscosity-ratio systems, fluid flows readily from the central region towards the pressure minimum (and the relative motion of the bulbous end is slower) causing a local minimum in the drop radius. The ends 'pinch-off' owing to flow away from the corresponding maximum in capillary pressure, which causes the 'neck' to become more pronounced and eventually 'pinch'. Notice that the higher curvature at the ends of very low-viscosity-ratio drops should lead to a rapid bulbing of the ends (i.e. high velocities near the end of the drop) followed by the pinching process just discussed. This is seen clearly for $\lambda=0.01$ in figure $9(a)$. 


\subsection{Numerical solution of the motion of an elongated drop via the boundary-integral method}

In order to test the qualitative description presented above and to understand more about the dependence of the dynamies on the initial shape and viscosity ratio, a numerical study of the interfacial-tension-driven motion of an elongated drop in a quiescent fluid is currently in progress using a boundary-integral method. Here we present calculations for the case $\lambda=1$ only in order to show that the numerical simulation agrees with the description of the physics presented in $\$ 5.1$.

The boundary-integral method has been applied to the low-Reynolds-number deformation of bubbles and drops in an external flow by Youngren \& Acrivos (1976), Rallison \& Acrivos (1978) and Rallison (1981). The technique is well suited to free-boundary problems since the interfacial velocities may be obtained directly, without the necessity of determining the velocity field in the entire flow domain.

Our overall objective is the examination of the dynamics of an initially extended liquid droplet that is suspended in a second immiscible fluid which is at rest at infinity. Inertial effects are negligible with respect to viscous effects provided $\rho \sigma l_{\mathrm{c}} / \mu^{2}(1+\lambda) \ll 1$, where $l_{\mathrm{c}}$ is some characteristic lengthscale of the deformed drop. As discussed specifically by Rallison \& Acrivos (1978), for the special case $\lambda=1$, the velocity field, non-dimensionalized with respect to $\sigma / \mu(1+\lambda)$, at any point, $x$ in the fluid domain, is given by

$$
u(x)=\frac{-1}{4 \pi} \int_{\mathrm{s}}\left[\frac{I}{\|x-\xi\|}+\frac{(x-\xi)(x-\xi)}{\|x-\xi\|^{3}}\right] \cdot n(\xi)\left[\nabla_{\mathrm{s}} \cdot n(\xi)\right] \mathrm{d} S(\xi),
$$

where $S$ represents the drop surface. Here, $\boldsymbol{n}$ is the unit outward normal from the drop surface and $\nabla_{\mathrm{s}} \cdot \boldsymbol{n}$ is the surface curvature. Thus, for the case when the drop and suspending fluids have the same viscosity, the velocity field may be thought of as generated by a 'membrane of Stokeslets distributed along the interface' with a density proportional to the local curvature. If we wish to follow the interface motion, (3) is used in conjunction with the kinematic condition, which may be stated symbolically as $\mathrm{d} S / \mathrm{d} t=\boldsymbol{u} \cdot \boldsymbol{n}$ for $\boldsymbol{x} \in S$ (time has been non-dimensionalized with respect to $l_{\mathrm{c}} \mu(1+\lambda) / \sigma$ ). As is evident from (3), the evolution of the drop shape is completely dependent on the initial drop geometry and the only role interfacial tension plays is to determine the characteristic velocity scale and, hence, the timescale of the relaxation/breakup process.

In this short discussion, we aim to demonstrate that the drop shape evolves in a manner representative of the 'end-pinching' phenomena and then examine the interior velocity field to see whether the behaviour is qualitatively consistent with the proposal of the preceding section. The details of the numerics and morecomprehensive results will be reported in a future communication. Here we simply note that the numerical scheme incorporates methods used for similar free-boundary problems by Rallison \& Acrivos (1978), Lee \& Leal (1982), Geller, Lee \& Leal (1986), and E. J. Hinch et al. (private communication). In the calculation reported below: the interface was subdivided into 24 elements; the interface shape was approximated by fitting a cubic spline to the collocation points; every few iterations the collocation points were evenly redistributed (based on arclength) along the interface; all integrals were evaluated using a Gauss quadrature scheme; and the drop volume, monitored as time progressed, was found to change by less than $1 \%$ over several hundred iterations.

Figure $15(a)$ represents the time evolution of a typical drop shape, with an initial 


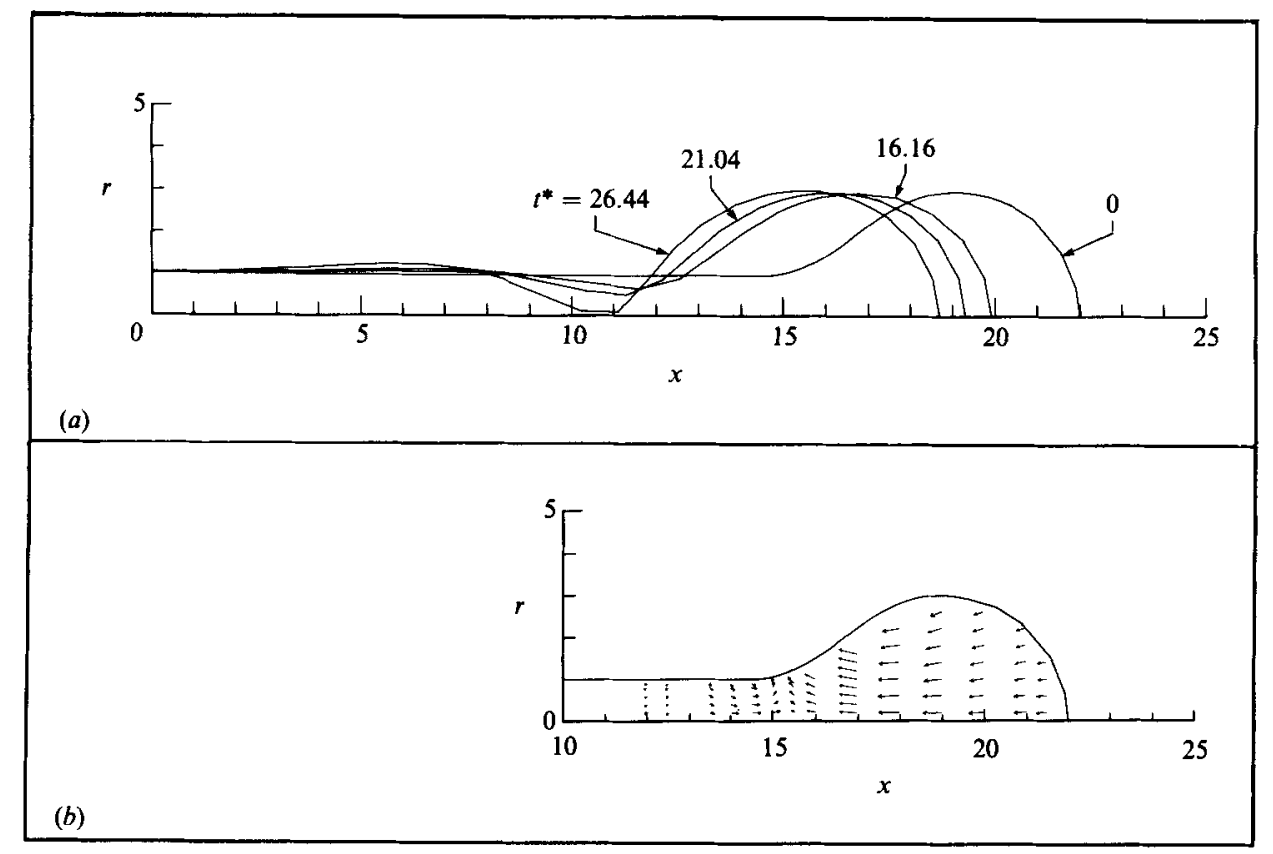

Figure 15. (a) Time evolution of an initially elongated drop suspended in an otherwise quiescent fluid, $t^{*}=l_{\mathrm{c}} \mu(1+\lambda) / \sigma$, where $l_{\mathrm{c}}$ is chosen as the initial radius at the centre of the drop and $\lambda=1$. (b) The velocity field in the drop for the initial shape shown in $(a)$.

elongation ratio $L / a=\mathbf{5 . 3}$, and an initial shape that can be approximated as a cylindrical midsection with a spherical end, the ratio of bulb to cylinder radius being $3: 1$. We have chosen the local radius as the characteristic lengthscale and $t^{*}$ is the dimensionless time that identifies evolution of the drop shape. We observe that the bulbous end translates towards the drop centre, a 'neck' slowly develops, and then the bulbous end rapidly begins to pinch-off. The evolution is very similar qualitatively to the many photographs presented above for $\lambda=O(1)$. In order to accurately describe the details of the pinch process, more points would have to be distributed along the interface to resolve the regions of high curvature. However, our goal is primarily to show that the elongated drop does undergo an 'end-pinching' process and that the interior velocity field is qualitatively consistent with the mechanism suggested in §5.1. For the latter purpose, it is sufficient to examine the initial velocity field at $t=\mathrm{O}^{+}$when the surface resolution is more than adequate. These results are shown in figure $15(b)$. We see that the end induces a strong, almost uniform flow toward the drop centre. Furthermore, there is indeed a flow, albeit weak, from the cylindrical region towards the pressure minimum, as was suggested in the qualitative discussion in \$5.1. It is this initial flux that leads to the development of a 'neck' and, consequently, a local pressure maximum, which will eventually result in drop fragmentation via 'end pinching' as depicted in figure 15(a). Hence, this example lends support to the qualitative explanation that we have presented to describe the relaxation/breakup process.

This work was supported by a grant from the Fluid Mechanics Program of the National Science Foundation. 


\section{REFERENCES}

Acrivos, A. 1983 The breakup of small drops and bubbles in shear flows. 4th Int Conf. on Physiochemical Hydrodynamics, Ann. N.Y. Acad. Sci. 404, 1-11.

Bentley, B. J. \& LEAL, L. G. $1986 a$ A computer-controlled four-roll mill for investigations of particle and drop dynamics in two-dimensional linear shear flows. J. Fluid Mech. 167, 219240.

Bentley, B. J. \& Leal, L. G. $1986 b$ An experimental investigation of drop deformation and breakup in steady two-dimensional linear flows. J. Fluid Mech. 167, 241-283.

Cox, R. G. 1969. The deformation of a drop in a general time-dependent fluid flow. J. Fluid Mech. 98, 305-328.

GeLler, A. S., LEe, S. H. \& Leat, L. G. 1986 The creeping motion of a spherical particle normal to a deformable interface. J. Fluid Mech. 169, 27-69.

Girault, H. H., Schiffrin, D. J. \& Smith, B. D. V. 1982 Drop image processing for surface and interfacial tension measurements. J. Electroanal. Chem. 137, 207-217.

Grradlt, H. H., Schiffrin, D. J. \& Smith, B. D. V. 1984 The measurement of interfacial tension of pendant drops using a video image profile digitizer. J. Colloid Interface Sci. 101, 257266.

GRACE, H.P. 1971 Dispersion phenomena in high viscosity immiscible fluid systems and application of static mixers as dispersion devices in such systems. Eng. Found. Res. Conference Mixing, 3rd Andover, N.H. Republished 1982 in Chem. Engng Commun. 14, 225-277.

HrNch, E. J. 1980 The evolution of slender inviscid drops in an axisymmetric straining flow. $J$. Fluid Mech, 101, 545-553.

Hinch, E. J. \& Acrivos, A. 1980 Long slender drops in a simple shear flow. J. Fluid Mech. 98 , 305-328.

LEe, S. H. \& LeAL, L. G. 1982 The motion of a sphere in the presence of a deformable interface. II. A numerical study of the translation of a sphere normal to an interface. J. Colloid Interface. Sci. 87, 81-106.

LEe, W.-K. \& FlUmerfelt, R. W. 1981 Instability of stationary and uniformly moving cylindrical fluid bodies. I. Newtonian systems, Intl J. Multiphase Flow 7, 363-383.

Lee, W.-K., YU, K.-L. \& Flumerfelt, R. W. 1981 Instability of stationary and uniformly moving cylindrical fluid bodies. II. Viscoelastic threads and experimental observations. Intl J. Multiphase Flow 7, 385-400.

Mikami, T., Cox, R. G. \& Mason, S. G. 1975 Breakup of extending liquid threads. Intl $J$. Multiphase Flow 2, 113-138.

RaLLison, J. M. 1980 Note on the time-dependent deformation of a viscous drop which is almost spherical. J. Fluid Mech. 98, 625-633.

RaLLison, J. W. 1981 A numerical study of the deformation and burst of a viscous drop in general shear flows. J. Fluid Mech. 109, 465-482.

RaLLison, J. M. 1984 The deformation of small viscous drops and bubbles in shear flows. Ann. Rev. Fluid Mech. 16, 45-66.

Rallison, J. M. \& ACRIvos, A. 1978 A numerical study of the deformation and burst of a viscous drop in an extensional flow. J. Fluid Mech. 89, 191-200.

RuMScheidT, F. D. \& Mason, S. G. 1961 Breakup of stationary liquid threads. J. Colloid Sci. 17, 260-269.

TAYLOR, G. I. 1932 The viscosity of a fluid containing small drops of another fluid. Proc. R. Soc. Lond. A 138, 41-48.

TAYLOR, G. I. 1934 The formation of emulsions in definable fields of flow. Proc. R. Soc. Lond. A 146, 501-523.

TAYLOR, G. I. 1964 Conical free surfaces and fluid interfaces. In Proc. Intl Congr. Appl. Mech., 11th, Munich, pp. 790-796.

Tомотіка, S. 1935 On the instability of a cylindrical thread of a viscous liquid surrounded by another viscous fluid. Proc. R. Soc. Lond. A 150, 322-337.

ТомотікA, S. 1936 Breaking up of a drop of viscous liquid immersed in another viscous fluid which is extending at a uniform rate. Proc. $R$. Soc. Lond. A 153, 302-318. 
Torza, S., Cox, R. G. \& Mason, S. G. 1972 Particle motions in sheared suspensions. XXVII. Transient and steady deformation and burst of liquid drops. J. Colloid Interface Sci. 38 , 395-411.

Youngren, G. K. \& Acrivos, A. 1976 On the shape of a gas bubble in a viscous extensional flow. J. Fluid Mech. 76, 433-442. 